\title{
High-order compact finite volume scheme for the 2D multi-term time fractional sub-diffusion equation
}

\author{
Baojin Su ${ }^{1}$ and Ziwen Jiang ${ }^{1 *}$
}

\section{"Correspondence:}

ziwjiang@126.com

'School of Mathematics and

Statistics, Shandong Normal

University, Jinan, Shandong, China

\begin{abstract}
Based on an $L 1$ interpolation operator, a new high-order compact finite volume scheme is derived for the $2 \mathrm{D}$ multi-term time fractional sub-diffusion equation. It is shown that the difference scheme is unconditionally convergent and stable in $L_{\infty}$-norm. The convergence order is $O\left(\tau^{2-\alpha}+h_{1}^{4}+h_{2}^{4}\right)$, where $\tau$ is the temporal step size and $h_{1}$ is the spatial step size in one direction, $h_{2}$ is the spatial step size in another direction. Two numerical examples are implemented, testifying to their efficiency and confirming their convergence order.
\end{abstract}

Keywords: 2D multi-term time fractional sub-diffusion equation; High-order compact finite volume scheme; Stable; Convergent

\section{Introduction}

The study of fractional partial differential equations has attracted many scholars' attention in recent decades $[27-29,32]$. Fractional differential equations are invoked in various scientific and engineering applications now. Physical phenomena in these fields, such as semiconductor, nuclear magnetic resonance, and signal processing, are successfully described by differential equations involving derivatives of fractional order [5, 6, 24, 25]. Moreover, some physical phenomena cannot be described by single-term time fractional partial differential equations, and they have to be described by multi-term time fractional partial differential equations [4]. For example, when modeling various types of viscoelastic damping one must use multi-term time fractional partial differential equations. Because the fractional integrals and derivatives satisfy the nonlocal properties, fractional-order partial differential equations are different from classical partial differential equations.

Many theoretical analyses about the multi-term time fractional partial differential equations have been carried out, but the analytic solutions cannot be obtained exactly in most fractional partial differential equations [10,23]. Other scholars have discussed the numerical solution of fractional partial differential equations by using different methods, such as finite difference method, compact finite difference method, finite element method, alternating direction implicit schemes, and so on $[1-3,7-9,11-13,15-17,19-22,26,30,34-$ $36,38-41,43-45,47,48,50,51,53,54]$. Agarwal et al. discussed the solvability a linear

(c) The Author(s) 2020. This article is licensed under a Creative Commons Attribution 4.0 International License, which permits use, sharing, adaptation, distribution and reproduction in any medium or format, as long as you give appropriate credit to the original author(s) and the source, provide a link to the Creative Commons licence, and indicate if changes were made. The images or other third party material in this article are included in the article's Creative Commons licence, unless indicated otherwise in a credit line to the material. If material is not included in the article's Creative Commons licence and your intended use is not permitted by statutory regulation or exceeds the permitted use, you will need to obtain permission directly from the copyright holder. To view a copy of this licence, visit http://creativecommons.org/licenses/by/4.0/. 
loaded integro-differential equation in an infinite three-dimensional domain [1]. Then, Agarwal et al. analyzed special functions of differential equations [2]. Alderremy et al. structured new models of the multi-space fractional Gardner equation [3]. Wang et al. considered some tractable cases of limit values in which either a difference of two ingredients or a difference equation is used coupled with the relevant functional equations to give rise to unexpected results [41]. El-Sayed et al. introduced a numerical technique for solving a class of multi-term variable-order fractional differential equations [11]. Chen and Liu structured a finite difference method for two-dimensional anomalous sub-diffusion equation, and they analyzed the stability and convergence of the scheme [7]. Yuste and Acedo found a finite difference method which can solve the time fractional diffusion equation by using a forward Euler scheme, and they discussed the stability and convergence of the scheme [48]. Ren and Sun proposed some efficient and stable numerical methods for multi-term time fractional sub-diffusion equations, and they proved that these methods are stable convergent in the sense of the maximum norm [30]. Liu has made a great contribution to fractional partial differential equations by using a finite difference method and discussed the stability and convergence by using a new energy method [19, 21]. Tadjeran et al. structured a second-order accurate numerical approximation for the fractional diffusion equation [36]. Sun et al. introduced a linearized second-order difference scheme for the nonlinear time fractional fourth-order reaction-diffusion equation [34]. Cui obtained finite difference schemes for the variable coefficients single- and multi-term time fractional diffusion equations [8]. To obtain higher order accuracy, a compact finite difference scheme is put forward. Wang et al. proposed compact difference schemes for the modified anomalous fractional sub-diffusion equation and the fractional diffusion-wave equation [44]. Cui structured a compact difference scheme for time fractional fourth-order equation with first Dirichlet boundary condition and discussed the stability and convergence [9]. Gao and Liu proposed a compact difference scheme for fourth-order temporal multi-term fractional wave equations and obtained maximum error estimates [13]. Later, Ji and Sun provided a high-order compact finite difference scheme for the fractional subdiffusion equation [16]. Zhuang et al. structured a new solution of the implicit numerical methods for the anomalous sub-diffusion equation and a numerical method of the modified anomalous sub-diffusion equation with a nonlinear source term [20,53]. Liu et al. provided finite element approximation for a modified anomalous sub-diffusion equation and a finite element method for two-dimensional multi-term time fractional diffusion wave equations [22, 45]. Langlands et al. introduced an implicit solution method for the fractional diffusion equation and proved the accuracy and stability of the scheme [17]. Wang et al. proposed a high-order compact ADI method for two-dimensional fractional convection sub-diffusion equations with Neumann boundary conditions and proved its unconditional stability and global convergence to be $O\left(\tau^{2-\alpha}+h^{4}\right)$ in the discrete L2 norm [43]. Zhang and Sun obtained alternating direction implicit schemes for the two-dimensional fractional sub-diffusion equation, and they proved that schemes are unconditionally stable and the numerical solution converges in the maximum norm [50]. Yang et al. provided a variational iteration method for solving multi-order fractional differential equations [47]. Furthermore, Zhuang et al. structured an implicit numerical method for the nonlinear fractional reaction sub-diffusion process, and they proved that the scheme was conditionally stable using the Fourier method [54]. Later, Zhang et al. proposed a CrankNicolson type difference scheme for the sub-diffusion equation which has a variable time 
and proved its unconditional stability in the maximum norm [51]. Tuan et al. analyzed a backward problem for fractional diffusion equation with Riemann-Liouville derivative [40]. Tuan et al. solved final value problem for nonlinear time fractional reaction-diffusion equation with discrete data [38]. Tuan et al. handled a terminal value problem for a generalization of fractional diffusion equation with hyper-Bessel operator [39]. Nguyen et al. settled a terminal value problem for time fractional diffusion equation by using regularization [26]. In addition, more researchers have found other numerical schemes such as the finite element method and others [12, 15, 18, 33, 35, 37, 46, 52]. Zhou et al. structured a weak Galerkin finite element method for multi-term time fractional diffusion equations [52]. Lin et al. obtained a separable freconditioner for time-space fractional Caputo-Riesz diffusion equations [18]. Sheng and Shen proposed a space-time Petrov-Galerkin spectral method for time fractional diffusion equation [33]. Wu and Zhou provided parareal algorithms with local time integrators for time fractional differential equations [46]. Tang et al. introduced energy dissipation theory and numerical stability for time fractional phase field equations [37]. Obviously, the study of the time fractional sub-diffusion equation, which included only one item time fractional order, is thorough. In fractional physics, especially diffusion movement, the concept of Brown movement is extended because of the generalization of Gauss probability function. The scope of nuclear magnetic resonance is expanding by added resolving power, so one item time fractional order cannot explain this kind of problem. Multi-term time fractional sub-diffusion equations play an important role in actual production and life.

The finite volume method is also called the control volume method. This method is an important approximation method. We usually mesh the space and there are nonrepetitive control volumes near each grid point. Then, we integrate the equations separately on each control volume. Finally, we approximate the first-order partial derivatives with the function values of nodes. Zhang and Jiang provided a compact finite volume method for one-dimensional Schrödinger equation [49]. Wang et al. proposed a compact finite volume scheme for one-dimensional parabolic equations with third boundary conditions, and proved that the scheme is unconditionally stable [42]. The finite volume method has obvious advantages of integral conservation [31].

Consider the following two-dimensional multi-term time fractional sub-diffusion equation with homogeneous source term on the computational domain $\Omega \times[0, T]$ :

$$
\begin{cases}{ }_{0}^{C} D_{t}^{\alpha} u(x, y, t)+{ }_{0}^{C} D_{t}^{\beta} u(x, y, t)=\Delta u(x, y, t)+f(x, y, t), & (x, y) \in \Omega, t \in(0, T], \\ u(x, y, t)=\psi(x, y, t), & (x, y) \in \partial \Omega, t \in[0, T], \\ u(x, y, 0)=\varphi(x, y), & (x, y) \in \Omega,\end{cases}
$$

where $\Omega=\left(0, L_{1}\right) \times\left(0, L_{2}\right), \partial \Omega$ is the boundary, $0<\beta<\alpha<1$, and the operator ${ }_{0}^{C} D_{t}^{\alpha}$ denotes the Caputo fractional derivative of order $\alpha$ defined by

$$
{ }_{0}^{C} D_{t}^{\alpha} f(t)=\frac{1}{\Gamma(1-\alpha)} \int_{0}^{t} \frac{f^{\prime}(\xi)}{(t-\xi)^{\alpha}} d \xi
$$

$\Gamma(\cdot)$ is the gamma function, and $\psi(x, y, t), \varphi(x, y)$, and $f(x, y, t)$ are the known smooth functions, $\psi(x, y, 0)=\varphi(x, y)$.

For the numerical approximation, take three positive integers $M_{1}, M_{2}, N$, and let $h_{1}=$ $\frac{L_{1}}{M_{1}}, h_{2}=\frac{L_{2}}{M_{2}}, \tau=\frac{T}{N}$. Define $x_{i}=i h_{1}\left(0 \leq i \leq M_{1}\right), y_{j}=j h_{2}\left(0 \leq i \leq M_{2}\right), t_{n}=n \tau(0 \leq n \leq N)$, 
$\Omega_{h_{1}}=\left\{x_{i} \mid 0 \leq i \leq M_{1}\right\}, \Omega_{h_{2}}=\left\{y_{j} \mid 0 \leq i \leq M_{2}\right\}, \Omega_{\tau}=\left\{t_{n} \mid 0 \leq i \leq N\right\}$. Define $\bar{\Omega}_{h}=\Omega_{h_{1}} \times$ $\Omega_{h_{2}}, \Omega_{h}=\bar{\Omega}_{h} \cup \partial \Omega, \Omega_{h \tau}=\Omega_{h} \times \Omega_{\tau}$, then the computational domain $\Omega \times[0, T]$ is covered by $\Omega_{h \tau}$. Define primal partition $I_{h}$, grid element $I_{i j}=\left[x_{i}, x_{i+1}\right] \times\left[y_{j}, y_{j+1}\right]\left(0 \leq i \leq M_{1}-1,0 \leq j \leq\right.$ $\left.M_{2}-1\right)$. Define dual partition $I_{h}^{*}, I_{0 j}^{*}=\left[0, x_{\frac{1}{2}}\right] \times\left[y_{j-\frac{1}{2}}, y_{j+\frac{1}{2}}\right], I_{0 i}^{*}=\left[0, y_{\frac{1}{2}}\right] \times\left[x_{i-\frac{1}{2}}, x_{i+\frac{1}{2}}\right], I_{i j p}^{*}=$ $\left[x_{i-\frac{1}{2}}, x_{i+\frac{1}{2}}\right] \times\left[y_{j-\frac{1}{2}}, y_{j+\frac{1}{2}}\right]\left(1 \leq i \leq M_{1}-1,1 \leq j \leq M_{2}-1\right), I_{j M_{1}}^{*}=\left[y_{j-\frac{1}{2}}, y_{j+\frac{1}{2}}\right] \times\left[x_{M_{1}-\frac{1}{2}}, L_{1}\right]$, $I_{i M_{2}}^{*}=\left[x_{i-\frac{1}{2}}, x_{i+\frac{1}{2}}\right] \times\left[x_{M_{2}-\frac{1}{2}}, L_{2}\right]$. Suppose that $u=\left\{u_{i, j}^{n} \mid 0 \leq i \leq M_{1}, 0 \leq j \leq M_{2}, 0 \leq n \leq N\right\}$ is a grid function on $\Omega_{h \tau}$. For every grid function $u$, we define the following notations:

$$
\delta_{x} u_{i-\frac{1}{2}, j}^{n}=\frac{1}{h_{1}}\left(u_{i, j}^{n}-u_{i-1, j}^{n}\right), \quad \delta_{y} u_{i, j-\frac{1}{2}}^{n}=\frac{1}{h_{2}}\left(u_{i, j}^{n}-u_{i, j-1}^{n}\right) .
$$

By using an $L 1$ interpolation operator and a compact operator, we can construct a highorder compact finite scheme and establish the corresponding error estimate. The remainder of the article is arranged as follows. In Sect. 2, the compact finite volume scheme is derived. In Sect. 3, the existence and uniqueness, stability and convergence of the finite volume scheme are proved. In Sect. 4, two numerical examples are given to demonstrate the theoretical results. Finally, we obtain a brief conclusion.

\section{The derivation of the compact finite volume scheme}

To construct the compact finite volume scheme, we introduce some important lemmas.

Lemma 1 If $g(x) \in C^{3}\left[0, L_{1}\right]$ and $x_{i+\frac{1}{2}}=\left(i+\frac{1}{2}\right) h_{1}, 0 \leq i \leq M_{1}-1$, then

$$
\int_{x_{i-\frac{1}{2}}}^{x_{i+\frac{1}{2}}} g(x) d x=\frac{h_{1}}{24}\left[g\left(x_{i-1}\right)+22 g\left(x_{i}\right)+g\left(x_{i+1}\right)\right]+C_{1} h_{1}^{4},
$$

where

$$
C_{1}=\int_{-\frac{1}{2}}^{\frac{1}{2}} \frac{g^{3}\left(\theta_{1}\right)}{6} \xi\left(\xi^{2}-1\right) d \xi, \quad \theta_{1} \in\left(x_{i-\frac{1}{2}}, x_{i+\frac{1}{2}}\right) .
$$

Especially,

$$
\int_{0}^{x_{1}} \frac{h_{2}}{2} g(x) d x=\frac{h_{1}}{3} g\left(x_{0}\right)+\frac{5 h_{1}}{24} g\left(x_{1}\right)-\frac{h_{1}}{24} g\left(x_{2}\right)+O\left(h_{1}^{4}\right)
$$

and

$$
\int_{x_{M_{1}-\frac{1}{2}}}^{x_{M_{1}}} g(x) d x=-\frac{h_{1}}{24} g\left(x_{M_{1}-2}\right)+\frac{5 h_{1}}{24} g\left(x_{M_{1}-1}\right)+\frac{h_{1}}{3} g\left(x_{M_{1}}\right)+O\left(h_{1}^{4}\right) .
$$

Similarly, if $w(y) \in C^{3}\left[0, L_{2}\right]$, we obtain that

$$
\int_{y_{j-\frac{1}{2}}}^{y_{j+\frac{1}{2}}} w(x) d x=\frac{h_{2}}{24}\left[w\left(y_{j-1}\right)+22 w\left(y_{j}\right)+w\left(y_{j+1}\right)\right]+C_{2} h_{2}^{4},
$$

where

$$
C_{2}=\int_{-\frac{1}{2}}^{\frac{1}{2}} \frac{w^{3}\left(\theta_{2}\right)}{6} \xi\left(\xi^{2}-1\right) d \xi, \quad \theta_{2} \in\left(y_{j-\frac{1}{2}}, y_{j+\frac{1}{2}}\right) .
$$


Especially,

$$
\int_{0}^{y_{1}} \frac{1}{2} w(y) d y=\frac{h_{2}}{3} w\left(y_{0}\right)+\frac{5 h_{2}}{24} w\left(y_{1}\right)-\frac{h_{2}}{24} w\left(y_{2}\right)+O\left(h_{2}^{4}\right)
$$

and

$$
\int_{y_{M_{2}-\frac{1}{2}}}^{y_{M_{2}}} w(y) d y=-\frac{h_{2}}{24} w\left(y_{M_{2}-2}\right)+\frac{5 h_{2}}{24} w\left(y_{M_{2}-1}\right)+\frac{h_{2}}{3} w\left(y_{M_{2}}\right)+O\left(h_{2}^{4}\right) .
$$

Proof We only prove the first integral approximation. The proof process of the second integral approximation is similar to the first one. We use Lagrange interpolation to approximate $g(x)$ at the points $\left(x_{i-1}, g\left(x_{i-1}\right)\right),\left(x_{i}, g\left(x_{i}\right)\right)$, and $\left(x_{i+1}, g\left(x_{i+1}\right)\right)$. We can obtain that

$$
g(x)=L_{2}(x)+R_{2}(x)
$$

where

$$
\begin{aligned}
L_{2}(x)= & \frac{\left(x-x_{i}\right)\left(x-x_{i+1}\right)}{\left(x_{i-1}-x_{i}\right)\left(x_{i-1}-x_{i+1}\right)} g\left(x_{i-1}\right)+\frac{\left(x-x_{i-1}\right)\left(x-x_{i+1}\right)}{\left(x_{i}-x_{i-1}\right)\left(x_{i}-x_{i+1}\right)} g\left(x_{i}\right) \\
& +\frac{\left(x-x_{i-1}\right)\left(x-x_{i}\right)}{\left(x_{i+1}-x_{i-1}\right)\left(x_{i+1}-x_{i}\right)} g\left(x_{i+1}\right)
\end{aligned}
$$

and

$$
R_{2}(x)=\frac{g^{3}\left(\theta_{1}\right)}{6}\left(x-x_{i-1}\right)\left(x-x_{i}\right)\left(x-x_{i+1}\right), \quad \theta_{1} \in\left(x_{i-\frac{1}{2}}, x_{i+\frac{1}{2}}\right) .
$$

Integrating $g(x)$ by parts, we have

$$
\int_{x_{i-\frac{1}{2}}}^{x_{i+\frac{1}{2}}} g(x) d x=\int_{x_{i-\frac{1}{2}}}^{x_{i+\frac{1}{2}}}\left(L_{2}(x)+R_{2}(x)\right) d x .
$$

To obtain the approximation order, we use substitution to simplify integral terms. For example, let $\xi=\frac{x-i h_{1}}{h_{1}}$, so the integral terms can be simplified into

$$
L_{2}(\xi)=\frac{1}{2} \xi(\xi-1) g\left(x_{i-1}\right)-\left(\xi^{2}-1\right) g\left(x_{i}\right)+\frac{1}{2} \xi(\xi+1) g\left(x_{i+1}\right)
$$

and

$$
R_{2}(\xi)=\frac{g^{3}\left(\theta_{1}\right) h_{1}^{3}}{6} \xi\left(\xi^{2}-1\right) .
$$

Then

$$
\int_{x_{i-\frac{1}{2}}}^{x_{i+\frac{1}{2}}} L_{2}(x) d x=h_{1} \int_{-\frac{1}{2}}^{\frac{1}{2}} L_{2}(\xi) d \xi=\frac{h_{1}}{24}\left(g\left(x_{i-1}\right)+22 g\left(x_{i}\right)+g\left(x_{i+1}\right)\right)
$$

and

$$
\int_{x_{i-\frac{1}{2}}}^{x_{i+\frac{1}{2}}} R_{2}(x) d x=h_{1} \int_{-\frac{1}{2}}^{\frac{1}{2}} R_{2}(\xi) d \xi=h_{1}^{4} \int_{-\frac{1}{2}}^{\frac{1}{2}} \frac{g^{3}\left(\theta_{1}\right)}{6} \xi\left(\xi^{2}-1\right) d \xi .
$$


Following the proof process of the first conclusion, we easily obtain the second conclusion.

Define a grid function space $U_{h_{1}}=\left\{g \mid g=\left(g_{0}, g_{1}, \ldots, g_{M_{1}}\right)\right\}$. For every $g \in U_{h_{1}}$, define the integral operator as follows:

$$
\left(S_{x} g\right)_{i}=\frac{h_{1}}{24}\left(g_{i-1}+22 g_{i}+g_{i+1}\right), \quad\left(1 \leq i \leq M_{1}-1\right)
$$

with $\left(S_{x} g\right)_{0}=\frac{h_{1}}{3} g_{0}+\frac{5 h_{1}}{24} g_{1}-\frac{h_{1}}{24} g_{2},\left(S_{x} g\right)_{M_{1}}=-\frac{h_{1}}{24} g_{M_{1}-2}+\frac{5 h_{1}}{24} g_{M_{1}-1}+\frac{h_{1}}{3} g_{M_{1}}$.

Similarly, for every $w \in U_{h_{2}}$, define the integral operator as follows:

$$
\left(S_{y} w\right)_{j}=\frac{h_{2}}{24}\left(w_{j-1}+22 w_{j}+w_{j+1}\right), \quad\left(1 \leq j \leq M_{2}-1\right)
$$

with $\left(S_{y} w\right)_{0}=\frac{h_{2}}{3} w_{0}+\frac{5 h_{2}}{24} w_{1}-\frac{h_{2}}{24} w_{2},\left(S_{y} w\right)_{M_{2}}=-\frac{h_{2}}{24} w_{M_{2}-2}+\frac{5 h_{2}}{24} w_{M_{2}-1}+\frac{h_{2}}{3} w_{M_{2}}$.

Lemma 2 If $g(x) \in C^{5}\left[0, L_{1}\right]$ and $x_{i+\frac{1}{2}}=\left(i+\frac{1}{2}\right) h_{1}, 0 \leq i \leq M_{1}-1$, then

$$
\frac{1}{24}\left[g^{\prime}\left(x_{i-\frac{3}{2}}\right)+22 g^{\prime}\left(x_{i-\frac{1}{2}}\right)+g^{\prime}\left(x_{i+\frac{1}{2}}\right)\right]=\frac{1}{h_{1}}\left[g\left(x_{i}\right)-g\left(x_{i-1}\right)\right]+C_{1} h_{1}^{4},
$$

where

$$
\begin{aligned}
C_{1}= & \int_{0}^{1}\left\{\frac{1}{144}\left[g^{(5)}\left(x_{i-\frac{1}{2}}-\lambda h_{1}\right)+g^{(5)}\left(x_{i-\frac{1}{2}}+\lambda h_{1}\right)\right](1-\lambda)^{3}\right. \\
& \left.-\frac{1}{48}\left[g^{(5)}\left(x_{i-\frac{1}{2}}-\frac{\lambda}{2} h_{1}\right)+g^{(5)}\left(x_{i-\frac{1}{2}}+\frac{\lambda}{2} h_{1}\right)\right](1-\lambda)^{4}\right\} d \lambda .
\end{aligned}
$$

Especially,

$$
3 g^{\prime}\left(x_{\frac{1}{2}}\right)-5 g^{\prime}\left(x_{\frac{3}{2}}\right)+4 g^{\prime}\left(x_{\frac{5}{2}}\right)-g^{\prime}\left(x_{\frac{7}{2}}\right)=\frac{1}{h_{1}}\left[g\left(x_{1}\right)-g\left(x_{0}\right)\right]+O\left(h_{1}^{4}\right)
$$

and

$$
-g^{\prime}\left(x_{M_{1}-\frac{7}{2}}\right)+4 g^{\prime}\left(x_{M_{1}-\frac{5}{2}}\right)-5 g^{\prime}\left(x_{M_{1}-\frac{3}{2}}\right)+3 g^{\prime}\left(x_{M_{1-\frac{1}{2}}}\right)=\frac{1}{h_{1}}\left[g\left(x_{M_{1}}\right)-g\left(x_{M_{1}-1}\right)\right]+O\left(h_{1}^{4}\right) \text {. }
$$

Similarly, if $w(y) \in C^{5}\left[0, L_{2}\right]$ and $y_{j+\frac{1}{2}}=\left(j+\frac{1}{2}\right) h_{2}, 0 \leq i \leq M_{2}-1$, then

$$
\frac{1}{24}\left[w^{\prime}\left(y_{j-\frac{3}{2}}\right)+22 w^{\prime}\left(y_{j-\frac{1}{2}}\right)+w^{\prime}\left(y_{j+\frac{1}{2}}\right)\right]=\frac{1}{h_{2}}\left[w\left(y_{j}\right)-w\left(y_{j-1}\right)\right]+C_{2} h_{2}^{4},
$$

where

$$
\begin{aligned}
C_{2}= & \int_{0}^{1}\left\{\frac{1}{144}\left[w^{(5)}\left(y_{j-\frac{1}{2}}-\lambda h_{2}\right)+w^{(5)}\left(y_{j-\frac{1}{2}}+\lambda h_{2}\right)\right](1-\lambda)^{3}\right. \\
& \left.-\frac{1}{48}\left[w^{(5)}\left(y_{j-\frac{1}{2}}-\frac{\lambda}{2} h_{2}\right)+w^{(5)}\left(y_{j-\frac{1}{2}}+\frac{\lambda}{2} h_{2}\right)\right](1-\lambda)^{4}\right\} d \lambda .
\end{aligned}
$$


Especially,

$$
3 w^{\prime}\left(y_{\frac{1}{2}}\right)-5 w^{\prime}\left(y_{\frac{3}{2}}\right)+4 w^{\prime}\left(y_{\frac{5}{2}}\right)-w^{\prime}\left(y_{\frac{7}{2}}\right)=\frac{1}{h_{2}}\left[w\left(y_{1}\right)-w\left(y_{0}\right)\right]+O\left(h_{2}^{4}\right)
$$

and

$$
\begin{aligned}
& -w^{\prime}\left(y_{M_{2}-\frac{7}{2}}\right)+4 w^{\prime}\left(y_{M_{2}-\frac{5}{2}}\right)-5 w^{\prime}\left(y_{M_{2}-\frac{3}{2}}\right)+3 w^{\prime}\left(y_{M_{2}-\frac{1}{2}}\right) \\
& =\frac{1}{h_{2}}\left[w\left(y_{M_{2}}\right)-w\left(y_{M_{2}-1}\right)\right]+O\left(h_{2}^{4}\right) .
\end{aligned}
$$

Proof We also only prove the first equation. Based on the Taylor expansion, we obtain the following equations:

$$
\begin{aligned}
g\left(x_{i}\right)= & g\left(x_{i-\frac{1}{2}}\right)+\frac{h_{1}}{2} g^{\prime}\left(x_{i-\frac{1}{2}}\right)+\frac{h_{1}^{2}}{8} g^{\prime \prime}\left(x_{i-\frac{1}{2}}\right)+\frac{h_{1}^{3}}{48} g^{(3)}\left(x_{i-\frac{1}{2}}\right) \\
+ & \frac{h_{1}^{4}}{384} g^{(4)}\left(x_{i-\frac{1}{2}}\right)+\frac{1}{24} \int_{x_{i-\frac{1}{2}}}^{x_{i}} g^{(5)}(s)\left(x_{i}-s\right)^{4} d s, \\
g\left(x_{i-1}\right)= & g\left(x_{i-\frac{1}{2}}\right)-\frac{h_{1}}{2} g^{\prime}\left(x_{i-\frac{1}{2}}\right)+\frac{h_{1}^{2}}{8} g^{\prime \prime}\left(x_{i-\frac{1}{2}}\right)-\frac{h_{1}^{3}}{48} g^{(3)}\left(x_{i-\frac{1}{2}}\right) \\
& +\frac{h_{1}^{4}}{384} g^{(4)}\left(x_{i-\frac{1}{2}}\right)+\frac{1}{24} \int_{x_{i-\frac{1}{2}}}^{x_{i-1}} g^{(5)}(s)\left(x_{i-1}-s\right)^{4} d s, \\
g^{\prime}\left(x_{i-\frac{3}{2}}\right)= & g^{\prime}\left(x_{i-\frac{1}{2}}\right)-h_{1} g^{\prime \prime}\left(x_{i-\frac{1}{2}}\right)+\frac{h_{1}^{2}}{2} g^{(3)}\left(x_{i-\frac{1}{2}}\right) \\
& -\frac{h_{1}^{3}}{6} g^{(4)}\left(x_{i-\frac{1}{2}}\right)+\frac{1}{6} \int_{x_{i-\frac{1}{2}}}^{x_{i-\frac{3}{2}}} g^{(5)}(s)\left(x_{i-\frac{3}{2}}-s\right)^{3} d s, \\
g^{\prime}\left(x_{i+\frac{1}{2}}\right)= & g^{\prime}\left(x_{i-\frac{1}{2}}\right)+h_{1} g^{\prime \prime}\left(x_{i-\frac{1}{2}}\right)+\frac{h_{1}^{2}}{2} g^{(3)}\left(x_{i-\frac{1}{2}}\right) \\
& +\frac{h_{1}^{3}}{6} g^{(4)}\left(x_{i-\frac{1}{2}}\right)+\frac{1}{6} \int_{x_{i-\frac{1}{2}}}^{x_{i+\frac{1}{2}}} g^{(5)}(s)\left(x_{i+\frac{1}{2}}-s\right)^{3} d s .
\end{aligned}
$$

Similar to Lemma 1, to obtain the approximation order, we use substitution to simplify integral remainder. For example, let $s=\left(i-\frac{1}{2}\right) h+\frac{\lambda}{2} h$, so the integral remainder of (2) can be simplified into

$$
\frac{h_{1}^{5}}{768} \int_{0}^{1} g^{(5)}\left(x_{i-\frac{1}{2}}+\frac{\lambda}{2} h\right)(1-\lambda)^{4} d \lambda \text {. }
$$

So, we can simplify the integral remainder of (2)-(5) by using the same way, then

$$
\begin{aligned}
\frac{1}{24}\left[g^{\prime}\left(x_{i-\frac{3}{2}}\right)+22 g^{\prime}\left(x_{i-\frac{1}{2}}\right)+g^{\prime}\left(x_{i+\frac{1}{2}}\right)\right]-\frac{1}{h_{1}}\left[g\left(x_{i}\right)-g\left(x_{i-1}\right)\right] \\
=\frac{h_{1}^{4}}{144} \int_{0}^{1}\left[g^{(5)}\left(x_{i-\frac{1}{2}}-\lambda h_{1}\right)+g^{(5)}\left(x_{i-\frac{1}{2}}+\lambda h_{1}\right)\right](1-\lambda)^{3} d \lambda \\
\quad-\frac{h_{1}^{4}}{48} \int_{0}^{1}\left[g^{(5)}\left(x_{i-\frac{1}{2}}-\frac{\lambda}{2} h_{1}\right)+g^{(5)}\left(x_{i-\frac{1}{2}}+\frac{\lambda}{2} h_{1}\right)\right](1-\lambda)^{4} d \lambda
\end{aligned}
$$




$$
\begin{aligned}
= & h_{1}^{4} \int_{0}^{1}\left\{\frac{1}{144}\left[g^{(5)}\left(x_{i-\frac{1}{2}}-\lambda h_{1}\right)+g^{(5)}\left(x_{i-\frac{1}{2}}+\lambda h_{1}\right)\right](1-\lambda)^{3}\right. \\
& \left.-\frac{1}{48}\left[g^{(5)}\left(x_{i-\frac{1}{2}}-\frac{\lambda}{2} h_{1}\right)+g^{(5)}\left(x_{i-\frac{1}{2}}+\frac{\lambda}{2} h_{1}\right)\right](1-\lambda)^{4}\right\} d \lambda .
\end{aligned}
$$

Similarly, we easily draw the second conclusion.

Define a fractional index grid function space $\bar{U}_{h_{1}}=\left\{g \mid g=\left(g_{\frac{1}{2}}, g_{\frac{3}{2}}, \ldots, g_{M_{1}-\frac{1}{2}}\right)\right\}$. For every $g_{i-\frac{1}{2}} \in \bar{U}_{h_{1}}$, define a compact operator as follows:

$$
\left(A_{x} g\right)_{i-\frac{1}{2}}=\frac{1}{24}\left(g_{i-\frac{3}{2}}+22 g_{i-\frac{1}{2}}+g_{i+\frac{1}{2}}\right) \quad\left(2 \leq i \leq M_{1}-1\right)
$$

with

$$
\begin{aligned}
& \left(A_{x} g\right)_{\frac{1}{2}}=3 g\left(x_{\frac{1}{2}}\right)-5 g\left(x_{\frac{3}{2}}\right)+4 g\left(x_{\frac{5}{2}}\right)-g\left(x_{\frac{7}{2}}\right), \\
& \left(A_{x} g\right)_{M_{1}-\frac{1}{2}}=-g\left(x_{M_{1}-\frac{7}{2}}\right)+4 g\left(x_{M_{1}-\frac{5}{2}}\right)-5 g\left(x_{M_{1-\frac{3}{2}}}\right)+3 g\left(x_{M_{1}-\frac{1}{2}}\right) .
\end{aligned}
$$

Similarly, define a fractional index grid function space $\bar{U}_{h_{2}}=\left\{w \mid w=\left(w_{\frac{1}{2}}, w_{\frac{3}{2}}, \ldots, w_{M_{2}-\frac{1}{2}}\right)\right\}$. For every $w_{j-\frac{1}{2}} \in \bar{U}_{h_{2}}$, define a compact operator as follows:

$$
\left(A_{y} w\right)_{j-\frac{1}{2}}=\frac{1}{24}\left(w_{j-\frac{3}{2}}+22 w_{j-\frac{1}{2}}+w_{j+\frac{1}{2}}\right) \quad\left(2 \leq j \leq M_{2}-1\right)
$$

with $\left(A_{y} w\right)_{\frac{1}{2}}=3 w\left(y_{\frac{1}{2}}\right)-5 w\left(y_{\frac{3}{2}}\right)+4 w\left(y_{\frac{5}{2}}\right)-w\left(y_{\frac{7}{2}}\right),\left(A_{y} w\right)_{M_{2}-\frac{1}{2}}=-w\left(y_{M_{2}-\frac{7}{2}}\right)+4 w\left(y_{M_{2}-\frac{5}{2}}\right)-$ $5 w\left(y_{M_{2}-\frac{3}{2}}\right)+3 w\left(y_{M_{2}-\frac{1}{2}}\right)$.

Lemma 3 ([14]) If $0<\alpha<1, g \in C^{2}\left[0, t_{n}\right]$, then

$$
\begin{aligned}
& \frac{1}{\Gamma(1-\alpha)} \int_{0}^{t_{n}} \frac{g^{\prime}(\xi)}{\left(t_{n}-\xi\right)^{\alpha}} d \xi \\
& \quad=\frac{\tau^{-\alpha}}{\Gamma(2-\alpha)}\left[a_{0}^{\alpha} g\left(t_{n}\right)-\sum_{k=1}^{n-1}\left(a_{n-k-1}^{\alpha}-a_{n-k}^{\alpha}\right) g\left(t_{k}\right)-a_{n-1}^{\alpha} g(0)\right]+R_{t_{n}}^{\alpha}
\end{aligned}
$$

and

$$
\left|R_{t_{n}}^{\alpha}\right| \leq \frac{1}{\Gamma(2-\alpha)}\left[\frac{1}{4}+\frac{\alpha}{(1-\alpha)(2-\alpha)}\right] \max _{0 \leq t \leq t_{n}}\left|g^{\prime \prime}(t)\right| \tau^{2-\alpha},
$$

where $a_{k}^{\alpha}=(k+1)^{1-\alpha}-k^{1-\alpha}$.

Define an $L 1$ approximation operator $D_{\tau}^{\alpha}$ as follows:

$$
\begin{aligned}
& D_{\tau}^{\alpha} g_{i j}^{n}=\frac{\tau^{-\alpha}}{\Gamma(2-\alpha)}\left[a_{0}^{\alpha} g\left(t_{n}\right)-\sum_{k=1}^{n-1}\left(a_{n-k-1}^{\alpha}-a_{n-k}^{\alpha}\right) g\left(t_{k}\right)-a_{n-1}^{\alpha} g(0)\right], \\
& 0 \leq i \leq M_{1}, \quad 0 \leq j \leq M_{2}, \quad 0 \leq n \leq N,
\end{aligned}
$$

where the definition of $a_{k}^{\alpha}$ is the same as in Lemma 3. 
Let us now construct a compact finite volume scheme for problem (1). On $\Omega_{h \tau}$, we now define the grid functions

$$
U_{i, j}^{n}=u\left(x_{i}, y_{j}, t_{n}\right), \quad f_{i, j}^{n}=f\left(x_{i}, y_{j}, t_{n}\right), \quad 0 \leq i \leq M_{1}, 0 \leq j \leq M_{2}, 0 \leq n \leq N .
$$

Suppose $u(x, y, t) \in C_{x, y, t}^{5,5,2}(\Omega \times[0, T])$. We consider equation (1) at the point $\left(x, y, t_{n}\right)$, and we can have

$$
\begin{aligned}
& { }_{0}^{C} D_{t}^{\alpha} u\left(x, y, t_{n}\right)+{ }_{0}^{C} D_{t}^{\beta} u\left(x, y, t_{n}\right)=\frac{\partial^{2} u\left(x, y, t_{n}\right)}{\partial x^{2}}+\frac{\partial^{2} u\left(x, y, t_{n}\right)}{\partial y^{2}}+f\left(x, y, t_{n}\right), \\
& (x, y) \in \Omega, \quad 1 \leq n \leq N .
\end{aligned}
$$

Integrating (6) on dual elements, we have

$$
\begin{aligned}
& \int_{x_{i-\frac{1}{2}}}^{x_{i+\frac{1}{2}}} \int_{y_{j-\frac{1}{2}}}^{y_{j+\frac{1}{2}}}\left({ }_{0}^{C} D_{t}^{\alpha} u\left(x, y, t_{n}\right)+{ }_{0}^{C} D_{t}^{\beta} u\left(x, y, t_{n}\right)\right) d y d x \\
& \quad=\int_{x_{i-\frac{1}{2}}}^{x_{i+\frac{1}{2}}} \int_{y_{j-\frac{1}{2}}}^{y_{j+\frac{1}{2}}}\left(\frac{\partial^{2} u\left(x, y, t_{n}\right)}{\partial x^{2}}+\frac{\partial^{2} u\left(x, y, t_{n}\right)}{\partial y^{2}}+f\left(x, y, t_{n}\right)\right) d y d x, \\
& 1 \leq i \leq M_{1}-1, \quad 1 \leq j \leq M_{2}-1, \quad 1 \leq n \leq N .
\end{aligned}
$$

We obtain that

$$
\begin{aligned}
& \int_{x_{i-\frac{1}{2}}}^{x_{i+\frac{1}{2}}} \int_{y_{j-\frac{1}{2}}}^{y_{j+\frac{1}{2}}}\left({ }_{0}^{C} D_{t}^{\alpha} u\left(x, y, t_{n}\right)+{ }_{0}^{C} D_{t}^{\beta} u\left(x, y, t_{n}\right)\right) d y d x \\
& =\int_{y_{j-\frac{1}{2}}}^{y_{j+\frac{1}{2}}}\left(\frac{\partial u\left(x_{i+\frac{1}{2}}, y, t_{n}\right)}{\partial x}-\frac{\partial u\left(x_{i-\frac{1}{2}}, y, t_{n}\right)}{\partial x}\right) d y \\
& \quad+\int_{x_{i-\frac{1}{2}}}^{x_{i+\frac{1}{2}}}\left(\frac{\partial u\left(x, y_{j+\frac{1}{2}}, t_{n}\right)}{\partial y}-\frac{\partial u\left(x, y_{j-\frac{1}{2}}, t_{n}\right)}{\partial y}\right) d x \\
& \quad+\int_{x_{i-\frac{1}{2}}}^{x_{i+\frac{1}{2}}} \int_{y_{j-\frac{1}{2}}}^{y_{j+\frac{1}{2}}} f\left(x, y, t_{n}\right) d y d x, \\
& 1 \leq i \leq M_{1}-1, \quad 1 \leq j \leq M_{2}-1, \quad 1 \leq n \leq N .
\end{aligned}
$$

By using Lemma 2, we get that

$$
\begin{gathered}
A_{x} A_{y} \int_{x_{i-\frac{1}{2}}}^{x_{i+\frac{1}{2}}} \int_{y_{j-\frac{1}{2}}}^{y_{j+\frac{1}{2}}}\left({ }_{0}^{C} D_{t}^{\alpha} u\left(x, y, t_{n}\right)+{ }_{0}^{C} D_{t}^{\beta} u\left(x, y, t_{n}\right)\right) d y d x \\
=A_{x} A_{y} \int_{y_{j-\frac{1}{2}}}^{y_{j+\frac{1}{2}}}\left(\frac{\partial u\left(x_{i+\frac{1}{2}}, y, t_{n}\right)}{\partial x}-\frac{\partial u\left(x_{i-\frac{1}{2}}, y, t_{n}\right)}{\partial x}\right) d y \\
\quad+A_{x} A_{y} \int_{x_{i-\frac{1}{2}}}^{x_{i+\frac{1}{2}}}\left(\frac{\partial u\left(x, y_{j+\frac{1}{2}}, t_{n}\right)}{\partial y}-\frac{\partial u\left(x, y_{j-\frac{1}{2}}, t_{n}\right)}{\partial y}\right) d x
\end{gathered}
$$




$$
\begin{gathered}
+A_{x} A_{y} \int_{x_{i-\frac{1}{2}}}^{x_{i+\frac{1}{2}}} \int_{y_{j-\frac{1}{2}}}^{y_{j+\frac{1}{2}}} f\left(x, y, t_{n}\right) d y d x, \\
1 \leq i \leq M_{1}-1, \quad 1 \leq j \leq M_{2}-1, \quad 1 \leq n \leq N .
\end{gathered}
$$

And then, we apply Lemma 1 to deal with spatial integral and use Lemma 3 to discretize the time fractional derivative. To optimize formula layout, we use the following notations:

$$
\begin{aligned}
& A_{1} u_{i j}^{n}=\frac{1}{576} S_{x} S_{y}\left(D_{\tau}^{\alpha} u_{i j}^{n}+D_{\tau}^{\beta} u_{i j}^{n}\right), \\
& A_{2} u_{i j}^{n}=\frac{1}{24} S_{y}\left(\delta_{x} u_{i+\frac{1}{2}, j}^{n}-\delta_{x} u_{i-\frac{1}{2}, j}^{n}\right), \\
& A_{3} u_{i j}^{n}=\frac{1}{24} S_{x}\left(\delta_{y} u_{i, j+\frac{1}{2}}^{n}-\delta_{y} u_{i, j-\frac{1}{2}}^{n}\right), \\
& A_{4} u_{i j}^{n}=\frac{1}{576} S_{x} S_{y} u_{i-1, j}^{n}+\frac{22}{576} S_{x} S_{y} u_{i j}^{n}+\frac{1}{576} S_{x} S_{y} u_{i+1, j}^{n} .
\end{aligned}
$$

We obtain

$$
\begin{aligned}
A_{1} & {\left[U_{i-1, j-1}^{n}+22 U_{i, j-1}^{n}+U_{i+1, j-1}^{n}+22 U_{i-1, j}^{n}+484 U_{i j}^{n}\right.} \\
& \left.+22 U_{i+1, j}^{n}+U_{i-1, j+1}^{n}+22 U_{i, j+1}^{n}+U_{i+1, j+1}^{n}\right] \\
= & A_{2}\left[U_{i, j-1}^{n}+22 U_{i, j}^{n}+U_{i, j+1}^{n}\right]+A_{3}\left[U_{i-1, j}^{n}+22 U_{i j}^{n}+U_{i+1, j}^{n}\right] \\
& +A_{4}\left[f_{i, j-1}^{n}+22 f_{i j}^{n}+f_{i, j+1}^{n}\right]+r_{i j}^{n}, \quad 1 \leq n \leq N,
\end{aligned}
$$

where $r_{i j}^{n}=C_{1}\left(\tau^{2-\alpha}\right)+C_{2}\left(h_{1}^{4}+h_{2}^{4}\right)$.

Notice that $u(x, y, t)=\psi(x, y, t)$ and $u(x, y, 0)=\varphi(x, y)$, so we have

$$
\begin{aligned}
& U_{i, j}^{0}=\varphi\left(x_{i}, y_{j}\right), \quad 1 \leq i \leq M_{1}-1,1 \leq j \leq M_{2}-1, \\
& U_{0, j}^{n}=\psi\left(0, y_{j}, t_{n}\right), \quad U_{L_{1}, j}^{n}=\psi\left(L_{1}, y_{j}, t_{n}\right), \\
& U_{i, 0}^{n}=\psi\left(x_{i}, 0, t_{n}\right), \quad U_{i, L_{2}}^{n}=\psi\left(x_{i}, L_{2}, t_{n}\right), \quad 0 \leq n \leq N .
\end{aligned}
$$

So, we leave out the infinitesimal and replace exact solution $U_{i, j}^{n}$ with numerical solution $u_{i, j}^{n}$, then we obtain that

$$
\begin{aligned}
& A_{1}[ u_{i-1, j-1}^{n}+22 u_{i, j-1}^{n}+u_{i+1, j-1}^{n}+22 u_{i-1, j}^{n}+484 u_{i j}^{n} \\
&\left.+22 u_{i+1, j}^{n}+u_{i-1, j+1}^{n}+22 u_{i, j+1}^{n}+u_{i+1, j+1}^{n}\right] \\
&= A_{2}\left[u_{i, j-1}^{n}+22 u_{i, j}^{n}+u_{i, j+1}^{n}\right]+A_{3}\left[u_{i-1, j}^{n}+22 u_{i j}^{n}+u_{i+1, j}^{n}\right] \\
&+A_{4}\left[f_{i, j-1}^{n}+22 f_{i j}^{n}+f_{i, j+1}^{n}\right], \quad 1 \leq n \leq N, \\
& u_{i, j}^{0}= \varphi\left(x_{i}, y_{j}\right), \quad 1 \leq i \leq M_{1}-1,1 \leq j \leq M_{2}-1, \\
& u_{0, j}^{n}=\psi\left(0, y_{j}, t_{n}\right), \quad u_{L_{1}, j}^{n}=\psi\left(L_{1}, y_{j}, t_{n}\right), \\
& u_{i, 0}^{n}=\psi\left(x_{i}, 0, t_{n}\right), \quad u_{i, L_{2}}^{n}=\psi\left(x_{i}, L_{2}, t_{n}\right), \quad 0 \leq n \leq N .
\end{aligned}
$$

These above equations are the compact finite volume scheme of question (1). 


\section{Analysis of the compact finite volume scheme}

In this section, we prove the existence and uniqueness, stability and convergence of the compact finite volume scheme.

First, we introduce the norms in the space $U_{h}$. Let $g=\left(g_{00}, g_{01}, \ldots, g_{M_{1} M_{2}}\right) \in U_{h}$, where $U_{h}=U_{h_{1}} \times U_{h_{2}}$. Denote

$$
\|g\|_{\infty}=\max _{0<i<M_{1}, 0<j<M_{2},}\left|g_{i j}\right| .
$$

Some important lemmas, which can be used in the following verifications, will be given.

Lemma 4 ([14]) Suppose $\alpha \in(0,1)$, and the definition of $a_{l}^{\alpha}$ is the same as in Lemma (3), $l=0,1, \ldots$, then

(I) $1=a_{0}^{\alpha}>a_{1}^{\alpha}>a_{2}^{\alpha}>\cdots>a_{l}^{\alpha} ; a_{l}^{\alpha} \rightarrow 0$, when $l \rightarrow \infty$;

(II) $(1-\alpha) l^{-\alpha}<a_{l-1}^{\alpha}<(1-\alpha)(l-1)^{-\alpha}, l \geq 1$.

Lemma 5 Let $p=\left(p_{00}, p_{01}, \ldots, p_{M_{1} M_{2}}\right) \in U_{h}, q=\left(q_{00}, q_{01}, \ldots, q_{M_{1} M_{2}}\right) \in U_{h}$, then

$$
\|p+q\|_{\infty} \leq\|p\|_{\infty}+\|q\|_{\infty} .
$$

Theorem 1 The solution of the compact finite volume scheme (13)-(15) is existent and unique.

Proof Let $u^{n}=\left(u_{00}^{n}, u_{01}^{n}, \ldots, u_{M_{1} M_{2}}^{n}\right) \in U_{h}$, and the numerical solution of $u^{0}$ can be obtained by (14). If the numerical solutions of $u^{0}, u^{1}, \ldots, u^{n-1}$ are existing and unique, we can obtain nonhomogeneous linear equations about $u^{n}$ from (13) and (15). If homogeneous linear equations only have a zero solution can be proved, we can prove that the existence and uniqueness of $u^{n}$ can be proved. Define $S_{\alpha}=\tau^{\alpha} \Gamma(2-\alpha), S_{\beta}=\tau^{\beta} \Gamma(2-\beta)$.

$$
\begin{aligned}
\left(\frac{S_{x} S_{y}}{576} S_{\alpha}\right. & \left.+\frac{S_{x} S_{y}}{576 S_{\beta}}\right) u_{i-1, j-1}^{n}+\left(\frac{22 S_{x} S_{y}}{576 S_{\alpha}}+\frac{22 S_{x} S_{y}}{576 S_{\beta}}\right) u_{i, j-1}^{n} \\
& +\left(\frac{S_{x} S_{y}}{576 S_{\alpha}}+\frac{S_{x} S_{y}}{576 S_{\beta}}\right) u_{i+1, j-1}^{n}+\left(\frac{22 S_{x} S_{y}}{576 S_{\alpha}}+\frac{22 S_{x} S_{y}}{576 S_{\beta}}\right) u_{i-1, j}^{n} \\
& +\left(\frac{484 S_{x} S_{y}}{576 S_{\alpha}}+\frac{484 S_{x} S_{y}}{576 S_{\beta}}\right) u_{i, j}^{n}+\left(\frac{22 S_{x} S_{y}}{576 S_{\alpha}}+\frac{22 S_{x} S_{y}}{576 S_{\beta}}\right) u_{i+1, j}^{n} \\
& +\left(\frac{S_{x} S_{y}}{576 S_{\alpha}}+\frac{S_{x} S_{y}}{576 S_{\beta}}\right) u_{i-1, j+1}^{n}+\left(\frac{22 S_{x} S_{y}}{576 S_{\alpha}}+\frac{22 S_{x} S_{y}}{576 S_{\beta}}\right) u_{i, j+1}^{n} \\
& +\left(\frac{S_{x} S_{y}}{576 S_{\alpha}}+\frac{S_{x} S_{y}}{576 S_{\beta}}\right) u_{i+1, j+1}^{n} \\
= & \frac{1}{24} S_{y}\left(\delta_{x} u_{i+\frac{1}{2}, j-1}^{n}-\delta_{x} u_{i-\frac{1}{2}, j-1}^{n}\right)+\frac{22}{24} S_{y}\left(\delta_{x} u_{i+\frac{1}{2}, j}^{n}-\delta_{x} u_{i-\frac{1}{2}, j}^{n}\right) \\
& +\frac{1}{24} S_{y}\left(\delta_{x} u_{i+\frac{1}{2}, j+1}^{n}-\delta_{x} u_{i-\frac{1}{2}, j+1}^{n}\right)+\frac{1}{24} S_{x}\left(\delta_{y} u_{i-1, j+\frac{1}{2}}^{n}-\delta_{y} u_{i-1, j-\frac{1}{2}}^{n}\right) \\
& +\frac{22}{24} S_{x}\left(\delta_{y} u_{i, j+\frac{1}{2}}^{n}-\delta_{y} u_{i, j-\frac{1}{2}}^{n}\right)+\frac{1}{24} S_{x}\left(\delta_{y} u_{i+1, j+\frac{1}{2}}^{n}-\delta_{y} u_{i+1, j-\frac{1}{2}}^{n}\right) \\
1 \leq i \leq & M_{1}-1, \quad 1 \leq j \leq M_{2}-1 . \\
u_{0}^{n}= & u_{M}^{n}=0 . \\
1 &
\end{aligned}
$$


What we should do next is to prove that (16) and (17) only have a zero solution. Equation (16) can be rewritten into

$$
\begin{aligned}
\left(\frac{S_{x} S_{y}}{576 S_{\alpha}}+\frac{S_{x} S_{y}}{576 S_{\beta}}\right) u_{i-1, j-1}^{n}+\left(\frac{22 S_{x} S_{y}}{576 S_{\alpha}}+\frac{22 S_{x} S_{y}}{576 S_{\beta}}+\frac{2 S_{y}}{24 h_{1}}\right) u_{i, j-1}^{n} \\
+\left(\frac{S_{x} S_{y}}{576 S_{\alpha}}+\frac{S_{x} S_{y}}{576 S_{\beta}}\right) u_{i+1, j-1}^{n}+\left(\frac{22 S_{x} S_{y}}{576 S_{\alpha}}+\frac{22 S_{x} S_{y}}{576 S_{\beta}}+\frac{2 S_{x}}{24 h_{2}}\right) u_{i-1, j}^{n} \\
+\left(\frac{484 S_{x} S_{y}}{576 S_{\alpha}}+\frac{484 S_{x} S_{y}}{576 S_{\beta}}+\frac{44 S_{y}}{24 h_{1}}+\frac{44 S_{x}}{24 h_{2}}\right) u_{i, j}^{n}+\left(\frac{22 S_{x} S_{y}}{576 S_{\alpha}}+\frac{22 S_{x} S_{y}}{576 S_{\beta}}+\frac{2 S_{x}}{24 h_{2}}\right) u_{i+1, j}^{n} \\
+\left(\frac{S_{x} S_{y}}{576 S_{\alpha}}+\frac{S_{x} S_{y}}{576 S_{\beta}}\right) u_{i-1, j+1}^{n}+\left(\frac{22 S_{x} S_{y}}{576 S_{\alpha}}+\frac{22 S_{x} S_{y}}{576 S_{\beta}}+\frac{2 S_{y}}{24 h_{1}}\right) u_{i, j+1}^{n} \\
+\left(\frac{S_{x} S_{y}}{576 S_{\alpha}}+\frac{S_{x} S_{y}}{576 S_{\beta}}\right) u_{i+1, j+1}^{n} \\
=\left(\frac{S_{y}}{24 h_{1}}+\frac{S_{x}}{24 h_{2}}\right) u_{i-1, j-1}^{n}+\left(\frac{S_{y}}{24 h_{1}}+\frac{S_{x}}{24 h_{2}}\right) u_{i+1, j-1}^{n}+\frac{22 S_{y}}{24 h_{1}} u_{i-1, j}^{n}+\frac{22 S_{y}}{24 h_{1}} u_{i+1, j}^{n} \\
\quad+\frac{22 S_{x}}{24 h_{2}} u_{i, j-1}^{n}+\frac{22 S_{x}}{24 h_{2}} u_{i, j+1}^{n}+\left(\frac{S_{y}}{24 h_{1}}+\frac{S_{x}}{24 h_{2}}\right) u_{i-1, j+1}^{n}+\left(\frac{S_{y}}{24 h_{1}}+\frac{S_{x}}{24 h_{2}}\right) u_{i+1, j+1}^{n} \\
1 \leq i \leq M_{1}-1, \quad 1 \leq j \leq M_{2}-1 .
\end{aligned}
$$

By using Lemma 5, equation mentioned above also can be transformed into

$$
\left(\frac{S_{x} S_{y}}{S_{\alpha}}+\frac{S_{x} S_{y}}{S_{\beta}}+\frac{2 S_{y}}{h_{1}}+\frac{2 S_{x}}{h_{2}}\right)\left\|u^{n}\right\|_{\infty} \leq\left(\frac{2 S_{y}}{h_{1}}+\frac{2 S_{x}}{h_{2}}\right)\left\|u^{n}\right\|_{\infty} .
$$

If the equation holds on, we can obtain that $\left\|u^{n}\right\|_{\infty}=0$. Furthermore, $u^{n}=0$.

According to the inductive principle, equations (13)-(15) have a unique solution.

Theorem 2 Suppose that the compact finite volume scheme (13)-(15) has a solution. We record it as $\left\{v_{i j}^{n} \mid 0 \leq i \leq M_{1}, 0 \leq j \leq M_{2}, 0 \leq n \leq N\right\}$, then

$$
\left\|v^{k}\right\|_{\infty} \leq\left\|v^{0}\right\|_{\infty}+c \max _{1 \leq m \leq k}\left\|f^{m}\right\|_{\infty}, \quad 1 \leq k \leq N
$$

where $c=\frac{1}{2} \max \left\{T^{\alpha} \Gamma(1-\alpha), T^{\beta} \Gamma(1-\beta)\right\},\left\|f^{m}\right\|_{\infty}=\max _{1 \leq i \leq M_{1}-1,1 \leq j \leq M_{2}-1}\left|f_{i j}^{m}\right|$.

Proof To optimize formula layout, we use the following notations:

$$
\begin{aligned}
& \delta u_{i j}^{n}=u_{i-1, j-1}^{n}+u_{i+1, j-1}^{n}+u_{i-1, j+1}^{n}+u_{i+1, j+1}^{n}, \\
& \delta_{j} u_{i j}^{n}=u_{i, j-1}^{n}+u_{i, j+1}^{n}, \\
& \delta_{i} u_{i j}^{n}=u_{i-1, j}^{n}+u_{i+1, j}^{n}, \\
& \delta_{\alpha} u_{i j}^{n}=\sum_{k=1}^{n-1}\left(a_{n-k-1}^{\alpha}-a_{n-k}^{\alpha}\right) u_{i, j}^{k}+a_{n-1}^{\alpha} u_{i, j}^{0}, \\
& \delta_{\beta} u_{i j}^{n}=\sum_{k=1}^{n-1}\left(a_{n-k-1}^{\beta}-a_{n-k}^{\beta}\right) u_{i, j}^{k}+a_{n-1}^{\beta} u_{i, j}^{0} .
\end{aligned}
$$


Equation (13) can be written into

$$
\begin{aligned}
\left(\frac{S_{x}}{576} S_{\alpha}\right. & \left.+\frac{S_{x} S_{y}}{576 S_{\beta}}\right) \delta v_{i j}^{n}+\left(\frac{484 S_{x} S_{y}}{576 S_{\alpha}}+\frac{484 S_{x} S_{y}}{576 S_{\beta}}+\frac{44 S_{y}}{24 h_{1}}+\frac{44 S_{x}}{24 h_{2}}\right) v_{i, j}^{n} \\
& +\left(\frac{22 S_{x} S_{y}}{576 S_{\alpha}}+\frac{22 S_{x} S_{y}}{576 S_{\beta}}+\frac{2 S_{y}}{24 h_{1}}\right) \delta_{j} v_{i j}^{n} \\
& +\left(\frac{22 S_{x} S_{y}}{576 S_{\alpha}}+\frac{22 S_{x} S_{y}}{576 S_{\beta}}+\frac{2 S_{x}}{24 h_{2}}\right) \delta_{i} v_{i j}^{n} \\
= & \frac{S_{x} S_{y}}{576 S_{\alpha}}\left(\delta_{\alpha} v_{i-1, j-1}^{n}+\delta_{\alpha} v_{i+1, j-1}^{n}+\delta_{\alpha} v_{i-1, j+1}^{n}+\delta_{\alpha} v_{i+1, j+1}^{n}\right) \\
& +\frac{S_{x} S_{y}}{576 S_{\beta}}\left(\delta_{\beta} v_{i-1, j-1}^{n}+\delta_{\beta} v_{i+1, j-1}^{n}+\delta_{\beta} v_{i-1, j+1}^{n}+\delta_{\beta} v_{i+1, j+1}^{n}\right) \\
& +\frac{22 S_{x} S_{y}}{576 S_{\alpha}}\left(\delta_{\alpha} v_{i, j-1}^{n}+\delta_{\alpha} v_{i, j+1}^{n}+\delta_{\alpha} v_{i-1, j}^{n}+\delta_{\alpha} v_{i+1, j}^{n}\right) \\
& +\frac{22 S_{x} S_{y}}{576 S_{\beta}}\left(\delta_{\beta} v_{i, j-1}^{n}+\delta_{\beta} v_{i, j+1}^{n}+\delta_{\beta} v_{i-1, j}^{n}+\delta_{\beta} v_{i+1, j}^{n}\right) \\
& +\frac{484 S_{x} S_{y}}{576 S_{\alpha}} \delta_{\alpha} v_{i j}^{n}+\frac{484 S_{x} S_{y}}{576 S_{\beta}} \delta_{\beta} v_{i j}^{n}+\left(\frac{S_{y}}{24 h_{1}}+\frac{S_{x}}{24 h_{2}}\right) \delta v_{i j}^{n} \\
& +\frac{22 S_{y}}{24 h_{1}} \delta_{i} v_{i j}^{n}+\frac{22 S_{x}}{24 h_{2}} \delta_{j} v_{i j}^{n}+\frac{1}{576} S_{x} S_{y} \delta f_{i j}^{n}+\frac{484}{576} S_{x} S_{y} f_{i, j}^{n} \\
& \frac{22 S_{x} S_{y}}{576} f_{i, j-1}^{n}\left(\delta_{j} f_{i j}^{n}+\delta_{j} f_{i j}^{n}\right) \quad 1 \leq n \leq N .
\end{aligned}
$$

By using Lemma 5, we can obtain

$$
\begin{aligned}
\left(\frac{S_{x} S_{y}}{S_{\alpha}}+\frac{S_{x} S_{y}}{S_{\beta}}+\frac{2 S_{y}}{h_{1}}+\frac{2 S_{x}}{h_{2}}\right)\left\|v^{n}\right\|_{\infty} \\
\leq \frac{S_{x} S_{y}}{S_{\alpha}}\left[\sum_{k=1}^{n-1}\left(a_{n-k-1}^{\alpha}-a_{n-k}^{\alpha}\right)\left\|v^{k}\right\|_{\infty}+a_{n-1}^{\alpha}\left\|v^{0}\right\|_{\infty}\right] \\
\quad+\frac{S_{x} S_{y}}{S_{\beta}}\left[\sum_{k=1}^{n-1}\left(a_{n-k-1}^{\beta}-a_{n-k}^{\beta}\right)\left\|v^{k}\right\|_{\infty}+a_{n-1}^{\beta}\left\|v^{0}\right\|_{\infty}\right] \\
+\left(\frac{2 S_{y}}{h_{1}}+\frac{2 S_{x}}{h_{2}}\right)\left\|v^{n}\right\|_{\infty}+S_{x} S_{y}\left\|^{n}\right\|_{\infty} \\
=\frac{S_{x} S_{y}}{S_{\alpha}}\left[\sum_{k=1}^{n-1}\left(a_{n-k-1}^{\alpha}-a_{n-k}^{\alpha}\right)\left\|v^{k}\right\|_{\infty}+a_{n-1}^{\alpha}\left\|v^{0}\right\|_{\infty}\right] \\
+\frac{S_{x} S_{y}}{S_{\beta}}\left[\sum_{k=1}^{n-1}\left(a_{n-k-1}^{\beta}-a_{n-k}^{\beta}\right)\left\|^{k}\right\|_{\infty}+a_{n-1}^{\beta}\left\|v^{0}\right\|_{\infty}\right] \\
+\left(\frac{2 S_{y}}{h_{1}}+\frac{2 S_{x}}{h_{2}}\right)\left\|v^{n}\right\|_{\infty}+S_{x} S_{y} \frac{a_{n-1}^{\alpha}}{S_{\alpha}} \cdot \frac{S_{\alpha}}{2 a_{n-1}^{\alpha}}\left\|f^{n}\right\|_{\infty} \\
+S_{x} S_{y} \frac{a_{n-1}^{\beta}}{S_{\beta}} \cdot \frac{S_{\beta}}{2 a_{n-1}^{\beta}\left\|f^{n}\right\|_{\infty} \cdot}
\end{aligned}
$$


By using Lemma 4, we can obtain

$$
\begin{aligned}
\frac{S_{\alpha}}{2 a_{n-1}^{\alpha}} & \leq \frac{\tau^{\alpha} \Gamma(2-\alpha)}{2(1-\alpha) n^{-\alpha}}=\frac{t_{n}^{\alpha} \Gamma(1-\alpha)}{2}, \\
\frac{S_{\beta}}{2 a_{n-1}^{\beta}} & \leq \frac{\tau^{\beta} \Gamma(2-\beta)}{2(1-\beta) n^{-\beta}}=\frac{t_{n}^{\beta} \Gamma(1-\beta)}{2} .
\end{aligned}
$$

Bring (20) and (21) into (19), then

$$
\begin{aligned}
& \left(\frac{S_{x} S_{y}}{S_{\alpha}}+\frac{S_{x} S_{y}}{S_{\beta}}\right)\left\|v^{n}\right\|_{\infty} \\
& \leq \sum_{k=1}^{n-1}\left[\frac{S_{x} S_{y}}{S_{\alpha}}\left(a_{n-k-1}^{\alpha}-a_{n-k}^{\alpha}\right)+\frac{S_{x} S_{y}}{S_{\beta}}\left(a_{n-k-1}^{\beta}-a_{n-k}^{\beta}\right)\right]\left\|v^{k}\right\|_{\infty} \\
& \quad+\left(S_{x} S_{y} \frac{a_{n-1}^{\alpha}}{S_{\alpha}}+S_{x} S_{y} \frac{a_{n-1}^{\beta}}{S_{\beta}}\right)\left(\left\|v^{0}\right\|_{\infty}+c\left\|f^{n}\right\|_{\infty}\right),
\end{aligned}
$$

where $c=\frac{1}{2} \max \left\{T^{\alpha} \Gamma(1-\alpha), T^{\beta} \Gamma(1-\beta)\right\}$.

We prove the establishment of (18) now.

According to (22), we can obtain that when $n=1$,

$$
\left(\frac{S_{x} S_{y}}{S_{\alpha}}+\frac{S_{x} S_{y}}{S_{\beta}}\right)\left\|v^{1}\right\|_{\infty} \leq\left(S_{x} S_{y} \frac{a_{0}^{\alpha}}{S_{\alpha}}+S_{x} S_{y} \frac{a_{0}^{\beta}}{S_{\beta}}\right)\left(\left\|v^{0}\right\|_{\infty}+c\left\|f^{1}\right\|_{\infty}\right)
$$

It also can be written as

$$
\left\|v^{1}\right\|_{\infty} \leq\left\|v^{0}\right\|_{\infty}+c\left\|f^{1}\right\|_{\infty} .
$$

So, when $k=1$, equation (18) is valid.

Suppose that equation (22) also satisfies conditions when $k=1,2, \ldots, n-1$. According to $(22)$, we can obtain

$$
\begin{aligned}
&\left(\frac{S_{x} S_{y}}{S_{\alpha}}+\frac{S_{x} S_{y}}{S_{\beta}}\right)\left\|v^{n}\right\|_{\infty} \\
& \leq \sum_{k=1}^{n-1}\left[\frac{S_{x} S_{y}}{S_{\alpha}}\left(a_{n-k-1}^{\alpha}-a_{n-k}^{\alpha}\right)\right. \\
&\left.\left.+\frac{S_{x} S_{y}}{S_{\beta}}\left(a_{n-k-1}^{\beta}-a_{n-k}^{\beta}\right)\right]\left(\left\|v^{0}\right\|_{\infty}+\max _{1 \leq m \leq k}\left\|f^{m}\right\|_{\infty}\right)\right) \\
&+\left(S_{x} S_{y} \frac{a_{n-1}^{\alpha}}{S_{\alpha}}+S_{x} S_{y} \frac{a_{n-1}^{\beta}}{S_{\beta}}\right)\left(\left\|v^{0}\right\|_{\infty}+c\left\|f^{n}\right\|_{\infty}\right) \\
& \leq\left\{\sum_{k=1}^{n-1}\left[\frac{S_{x} S_{y}}{S_{\alpha}}\left(a_{n-k-1}^{\alpha}-a_{n-k}^{\alpha}\right)+\frac{S_{x} S_{y}}{S_{\beta}}\left(a_{n-k-1}^{\beta}-a_{n-k}^{\beta}\right)\right]\right. \\
&\left.\left.+S_{x} S_{y} \frac{a_{n-1}^{\alpha}}{S_{\alpha}}+S_{x} S_{y} \frac{a_{n-1}^{\beta}}{S_{\beta}}\right\}\left(\left\|v^{0}\right\|_{\infty}+\max _{1 \leq m \leq n}\left\|f^{m}\right\|_{\infty}\right)\right) \\
&=\left(\frac{S_{x} S_{y}}{S_{\alpha}}+\frac{S_{x} S_{y}}{S_{\beta}}\right)\left(\left\|v^{0}\right\|_{\infty}+c\left\|f^{n}\right\|_{\infty}\right) .
\end{aligned}
$$


It is equivalent to

$$
\left\|v^{n}\right\|_{\infty} \leq\left\|v^{0}\right\|_{\infty}+c\left\|f^{n}\right\|_{\infty} .
$$

So, when $k=n$, equation (18) satisfies conditions.

According to the inductive principle, Theorem 2 holds.

Theorem 3 The solution of the compact finite volume scheme (13)-(15) is convergent. Suppose that $\left\{U_{i j}^{n} \mid 0 \leq i \leq M_{1}, 0 \leq j \leq M_{2}, 0 \leq n \leq N\right\}$ is the solution of equation (1) and $\left\{u_{i j}^{n} \mid 0 \leq i \leq M_{1}, 0 \leq j \leq M_{2}, 0 \leq n \leq N\right\}$ is the solution of the compact finite volume scheme (13)-(15). Define

$$
e_{i j}^{n}=U_{i j}^{n}-u_{i j}^{n}, \quad 0 \leq i \leq M_{1}, 0 \leq j \leq M_{2}, 0 \leq n \leq N,
$$

then

$$
\left\|e^{n}\right\|_{\infty} \leq c\left(\tau^{2-\alpha}+h_{1}^{4}+h_{2}^{4}\right), \quad 1 \leq n \leq N .
$$

Proof By subtracting equations (13)-(15) from the compact finite volume scheme (10)(12), we can obtain error equations:

$$
\begin{aligned}
\frac{1}{576} S_{x} & S_{y}\left(D_{\tau}^{\alpha} e_{i-1, j-1}^{n}+D_{\tau}^{\beta} e_{i-1, j-1}^{n}\right)+\frac{22}{576} S_{x} S_{y}\left(D_{\tau}^{\alpha} e_{i, j-1}^{n}+D_{\tau}^{\beta} e_{i, j-1}^{n}\right) \\
& +\frac{1}{576} S_{x} S_{y}\left(D_{\tau}^{\alpha} e_{i+1, j-1}^{n}+D_{\tau}^{\beta} e_{i+1, j-1}^{n}\right)+\frac{22}{576} S_{x} S_{y}\left(D_{\tau}^{\alpha} e_{i-1, j}^{n}+D_{\tau}^{\beta} e_{i-1, j}^{n}\right) \\
& +\frac{484}{576} S_{x} S_{y}\left(D_{\tau}^{\alpha} e_{i, j}^{n}+D_{\tau}^{\beta} e_{i, j}^{n}\right)+\frac{22}{576} S_{x} S_{y}\left(D_{\tau}^{\alpha} e_{i+1, j}^{n}+D_{\tau}^{\beta} e_{i+1, j}^{n}\right) \\
& +\frac{1}{576} S_{x} S_{y}\left(D_{\tau}^{\alpha} e_{i-1, j+1}^{n}+D_{\tau}^{\beta} e_{i-1, j+1}^{n}\right)+\frac{22}{576} S_{x} S_{y}\left(D_{\tau}^{\alpha} e_{i, j+1}^{n}+D_{\tau}^{\beta} e_{i, j+1}^{n}\right) \\
& +\frac{1}{576} S_{x} S_{y}\left(D_{\tau}^{\alpha} e_{i+1, j+1}^{n}+D_{\tau}^{\beta} e_{i+1, j+1}^{n}\right) \\
= & \frac{1}{24} S_{y}\left(\delta_{x} e_{i+\frac{1}{2}, j-1}^{n}-\delta_{x} e_{i-\frac{1}{2}, j-1}^{n}\right)+\frac{22}{24} S_{y}\left(\delta_{x} e_{i+\frac{1}{2}, j}^{n}-\delta_{x} e_{i-\frac{1}{2}, j}^{n}\right) \\
& +\frac{1}{24} S_{y}\left(\delta_{x} e_{i+\frac{1}{2}, j+1}^{n}-\delta_{x} e_{i-\frac{1}{2}, j+1}^{n}\right)+\frac{1}{24} S_{x}\left(\delta_{y} e_{i-1, j+\frac{1}{2}}^{n}-\delta_{y} e_{i-1, j-\frac{1}{2}}^{n}\right) \\
& +\frac{22}{24} S_{x}\left(\delta_{y} e_{i, j+\frac{1}{2}}^{n}-\delta_{y} e_{i, j-\frac{1}{2}}^{n}\right)+\frac{1}{24} S_{x}\left(\delta_{y} e_{i+1, j+\frac{1}{2}}^{n}-\delta_{y} e_{i+1, j-\frac{1}{2}}^{n}\right)+r_{i j}^{n}, \\
1 \leq & n \leq N, \quad \\
e_{i, j}^{0}= & 0, \quad 1 \leq i \leq M_{1}-1,1 \leq j \leq M_{2}-1, \\
e_{0, j}^{n}= & 0, \quad e_{L_{1}, j}^{n}=0, \\
e_{i, 0}^{n}= & 0, \quad e_{i, L_{2}}^{n}=0, \quad 0 \leq n \leq N .
\end{aligned}
$$

By using Theorem 2, we can obtain

$$
\left\|e^{n}\right\|_{\infty} \leq\left\|e^{0}\right\|_{\infty}+c \max _{1 \leq k \leq n}\left\|r^{k}\right\|_{\infty}, \quad 1 \leq n \leq N
$$

where $c=\frac{1}{2} \max \left\{T^{\alpha} \Gamma(1-\alpha), T^{\beta} \Gamma(1-\beta)\right\}$. 
Furthermore, it is easy to obtain that

$$
\left\|r^{k}\right\|_{\infty} \leq c_{1}\left(\tau^{2-\alpha}+h^{4}\right)
$$

So, the result

$$
\left\|e^{n}\right\|_{\infty} \leq c\left(\tau^{2-\alpha}+h_{1}^{4}+h_{2}^{4}\right), \quad 1 \leq n \leq N
$$

is proved.

\section{Numerical experiments}

We now present two numerical examples using the numerical methods as discussed above, where the errors involved are measured by comparing the numerical solutions with exact solutions. To simplify the calculation, let $h=h_{1}=h_{2}$.

Example 1 Let $\Omega=[0,1] \times[0.1], T=1$. In order to test the convergence of the finite volume scheme, we refer to the exact solution of problem (1) as

$$
u(x, y, t)=\frac{1}{3} t^{2} \sin (\pi x) \sin (\pi y)
$$

We can obtain the corresponding source term $f(x, y, t)$ and the initial and boundary conditions with $\alpha=0.4, \beta=0.2$, which are respectively

$$
\begin{aligned}
f(x, y, t)= & \frac{1}{3}\left(\frac{\Gamma(3)}{\Gamma(3-\alpha)} \sin (\pi x) \sin (\pi y) t^{2-\alpha}+\frac{\Gamma(3)}{\Gamma(3-\beta)} \sin (\pi x) \sin (\pi y) t^{2-\beta}\right. \\
& \left.+2 \pi^{2} t^{2} \sin (\pi x) \sin (\pi y)\right)
\end{aligned}
$$

and

$$
\psi(x, y, t)=0, \quad \varphi(x, y)=0 .
$$

Denote the maximum norm errors

$$
E_{\infty}\left(h_{1}, h_{2}, \tau\right)=\max _{1 \leq i \leq M_{1}, 1 \leq j \leq M_{2}}\left|U_{i j}^{N}-u_{i j}^{N}\right| .
$$

Tables 1 and 2 show the maximum norm errors and the convergence order of the finite volume scheme. In order to simplify the calculation, we let $h=h_{1}=h_{2}$. Suppose

$$
E_{\infty}(h, \tau)=O\left(h^{p}+\tau^{q}\right)
$$

If $\tau$ is small enough, then $E_{\infty}(h, \tau) \approx O\left(h^{p}\right)$. Consequently,

$$
\frac{E_{\infty}(2 h, \tau)}{E_{\infty}(h, \tau)} \approx 2^{p}, \quad \log 2 \frac{E_{\infty}(2 h, \tau)}{E_{\infty}(h, \tau)} \approx p .
$$


Table 1 Error behavior with Dirichlet boundary condition for $h=\frac{1}{1000}$

\begin{tabular}{lll}
\hline$\tau$ & $E_{\infty}(h, \tau)$ & Order $1 \approx$ \\
\hline $2^{-3}$ & $3.7015 \mathrm{e}-004$ & - \\
$2^{-4}$ & $1.3227 \mathrm{e}-004$ & 1.4846 \\
$2^{-5}$ & $4.5898 \mathrm{e}-005$ & 1.5270 \\
$2^{-6}$ & $1.5352 \mathrm{e}-005$ & 1.5799 \\
$2^{-7}$ & $5.0888 \mathrm{e}-006$ & 1.5931 \\
\hline
\end{tabular}

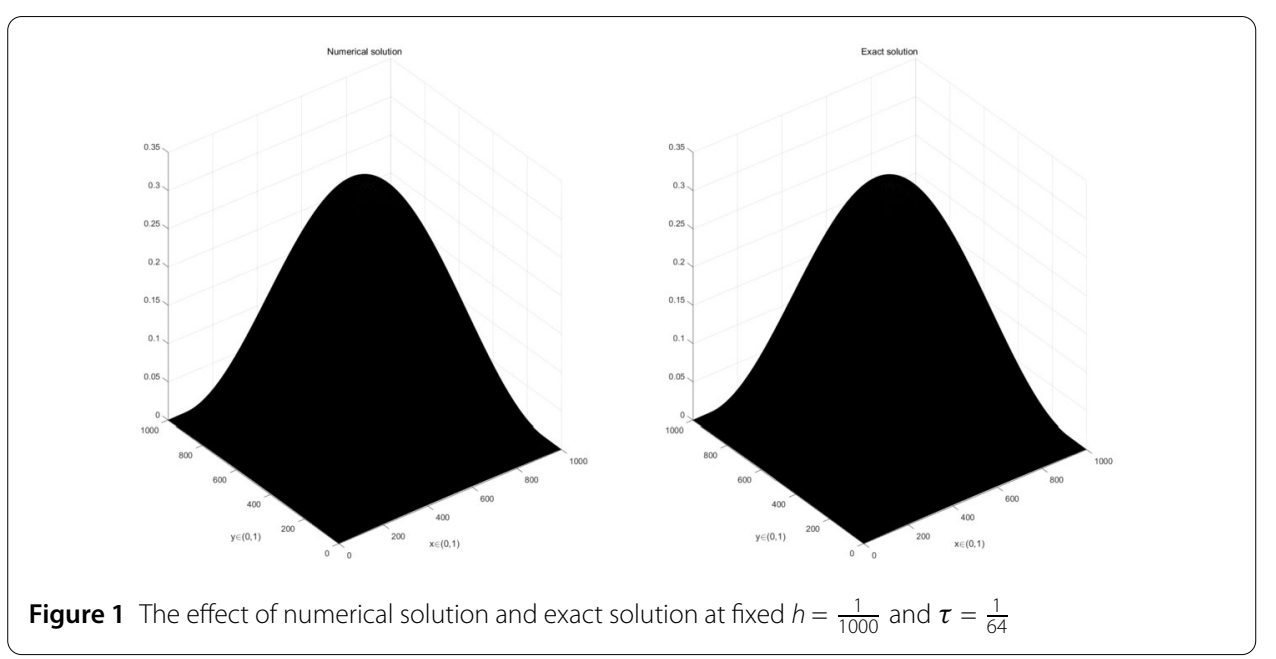

Table 2 Error behavior with Dirichlet boundary condition for $\tau=\frac{1}{1000}$

\begin{tabular}{llll}
\hline$h$ & $E_{\infty}(h, \tau)$ & Mass & Order2 $\approx$ \\
\hline $2^{-3}$ & $4.8386 \mathrm{e}-004$ & $1.3520 \mathrm{e}-001$ & - \\
$2^{-4}$ & $3.0735 \mathrm{e}-005$ & $1.3509 \mathrm{e}-001$ & 3.9767 \\
$2^{-5}$ & $1.9317 \mathrm{e}-006$ & $1.3487 \mathrm{e}-001$ & 3.9920 \\
$2^{-6}$ & $1.2167 \mathrm{e}-007$ & $1.3501 \mathrm{e}-001$ & 3.9892 \\
$2^{-7}$ & $7.8016 \mathrm{e}-009$ & $1.3551 \mathrm{e}-001$ & 3.9626 \\
\hline
\end{tabular}

So, $p$ is the convergence order with respect to the spatial step size. Similarly, we can obtain

$$
q \approx \log 2 \frac{E_{\infty}(h, 2 \tau)}{E_{\infty}(h, \tau)}
$$

for small enough $h$. Denote

$$
\begin{aligned}
& \operatorname{Order} 1=\log 2 \frac{E_{\infty}(h, 2 \tau)}{E_{\infty}(h, \tau)}, \\
& \operatorname{Order} 2=\frac{E_{\infty}(2 h, \tau)}{E_{\infty}(h, \tau)} .
\end{aligned}
$$

Define Mass $_{h}=\sum_{i=0}^{M_{1}} \sum_{j=0}^{M_{2}} u_{i j}^{N} h^{2}$. If under different meshes Mass $h$ keep about the same size, we can explain the integral conservation of the compact finite volume scheme.

We compare the exact solution and the numerical solution under different meshes. In Tables 1 and 2, temporal and spatial convergence orders are shown. We fix sufficiently small spatial step size $h=\frac{1}{1000}$ and vary the temporal step sizes. Table 1 lists the numerical results for different temporal step sizes. Similarly, we fix sufficiently small temporal step 

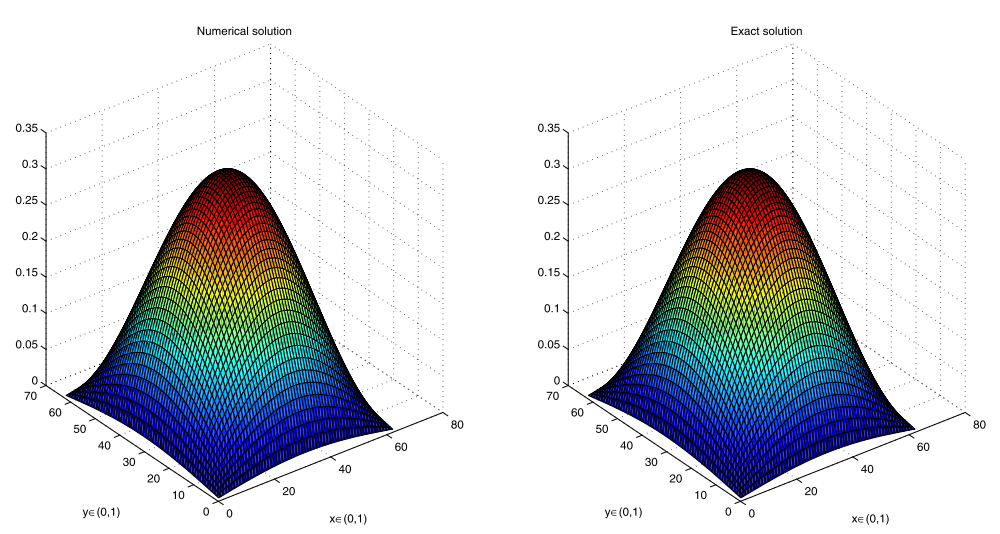

Figure 2 The effect of numerical solution and exact solution at fixed $\tau=\frac{1}{1000}$ and $h=\frac{1}{64}$

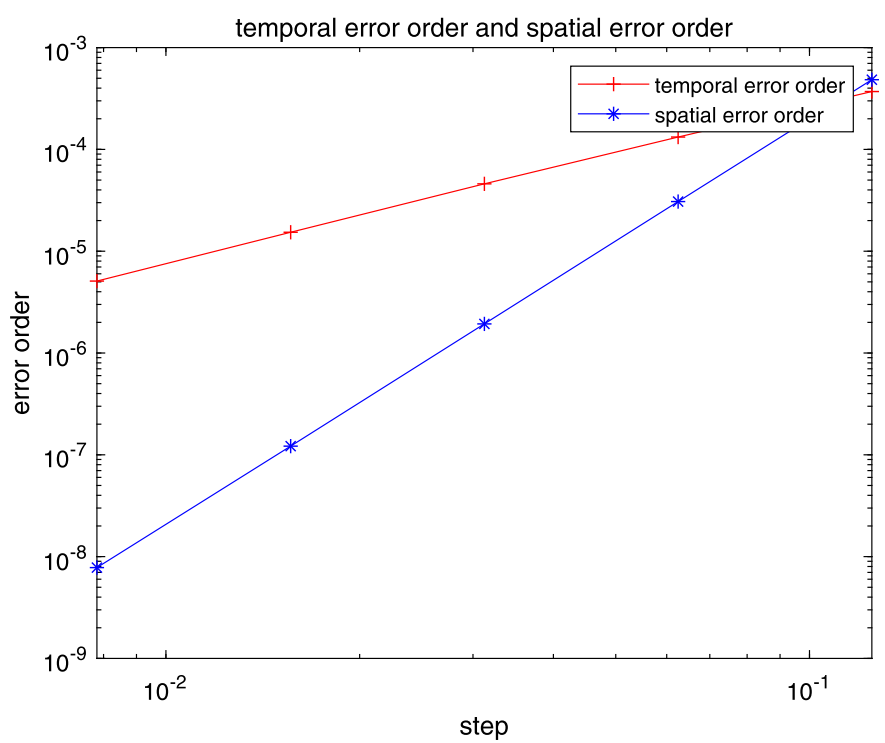

Figure 3 The red line represents temporal error order, the blue line represents spatial error order

sizes $\tau=\frac{1}{1000}$ and vary the spatial step sizes. Also, Table 2 lists the numerical results for different spatial step sizes.

In order to observe error orders more intuitively, we plot Fig. 3 about error orders, where slope represents the error order. We can observe that the temporal error order is about $2-\alpha$ and the spatial error order is about $h^{4}$.

Example 2 Let $\Omega=[0,1] \times[0.1], T=1$. As before, we refer to the exact solution of problem (1) as

$$
u(x, t)=t^{3}\left(-x+x^{2}\right)\left(-y+y^{2}\right) .
$$


It is also not difficult to obtain the corresponding source term $f(x, y, t)$ and the initial and boundary conditions with $\alpha=0.8, \beta=0.3$, which are respectively

$$
\begin{aligned}
f(x, y, t)= & \frac{\Gamma(4)}{\Gamma(4-\alpha)}\left(x-x^{2}\right)\left(y-y^{2}\right) t^{3-\alpha}+\frac{\Gamma(4)}{\Gamma(4-\beta)}\left(x-x^{2}\right)\left(y-y^{2}\right) t^{3-\beta} \\
& +2 t^{3}\left(y-y^{2}\right)+2 t^{3}\left(x-x^{2}\right)
\end{aligned}
$$

and

$$
\psi(x, y, t)=0, \quad \varphi(x, y)=0 .
$$

Like the previous example, we compute the maximum norm errors of the numerical solution to test the convergence of the compact finite volume scheme, which is defined the same as in Example 1. Denote

$$
\operatorname{Order} 1=\log 2 \frac{E_{\infty}(h, 2 \tau)}{E_{\infty}(h, \tau)}, \quad \operatorname{Order} 2=\frac{E_{\infty}(2 h, \tau)}{E_{\infty}(h, \tau)} .
$$

In Table 3, we compute the problem for a longer time by fixing $N=8,16,32,64,128$, and still choosing $h=\frac{1}{1000}$. And then, we compute the problem for a longer space by fixing $M=8,16,32,64,128$, and still choosing $\tau=\frac{1}{1000}$ in Table 4 .

As in Example 1, we plot Fig. 6 about error orders, where slope represents the error order. We can observe that the temporal error order is about $2-\alpha$ and the spatial error order is about $h^{4}$.

Table 3 Error behavior with Dirichlet boundary condition for $h=\frac{1}{1000}$

\begin{tabular}{lll}
\hline$\tau$ & $E_{\infty}(h, \tau)$ & Order $1 \approx$ \\
\hline $2^{-3}$ & $1.3640 \mathrm{e}-004$ & - \\
$2^{-4}$ & $6.1226 \mathrm{e}-005$ & 1.1556 \\
$2^{-5}$ & $2.6898 \mathrm{e}-005$ & 1.1866 \\
$2^{-6}$ & $1.1752 \mathrm{e}-005$ & 1.1945 \\
$2^{-7}$ & $5.1178 \mathrm{e}-006$ & 1.1993 \\
\hline
\end{tabular}
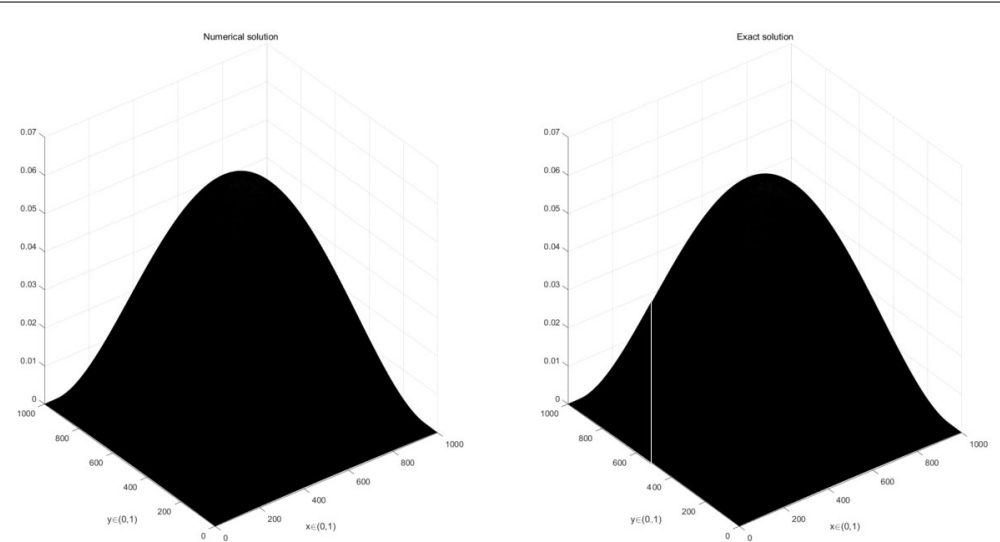

Figure 4 The effect of numerical solution and exact solution at fixed $h=\frac{1}{1000}$ and $\tau=\frac{1}{64}$ 
Table 4 Error behavior with Dirichlet boundary condition for $\tau=\frac{1}{1000}$

\begin{tabular}{llll}
\hline$h$ & $E_{\infty}(h, \tau)$ & Mass & Order2 $\approx$ \\
\hline $2^{-3}$ & $4.2467 \mathrm{e}-004$ & $2.6927 \mathrm{e}-002$ & - \\
$2^{-4}$ & $2.9237 \mathrm{e}-005$ & $2.7852 \mathrm{e}-002$ & 3.8605 \\
$2^{-5}$ & $2.0005 \mathrm{e}-006$ & $2.8751 \mathrm{e}-002$ & 3.8694 \\
$2^{-6}$ & $1.3253 \mathrm{e}-007$ & $2.8845 \mathrm{e}-002$ & 3.9160 \\
$2^{-7}$ & $8.5850 \mathrm{e}-009$ & $2.8021 \mathrm{e}-002$ & 3.9484 \\
\hline
\end{tabular}
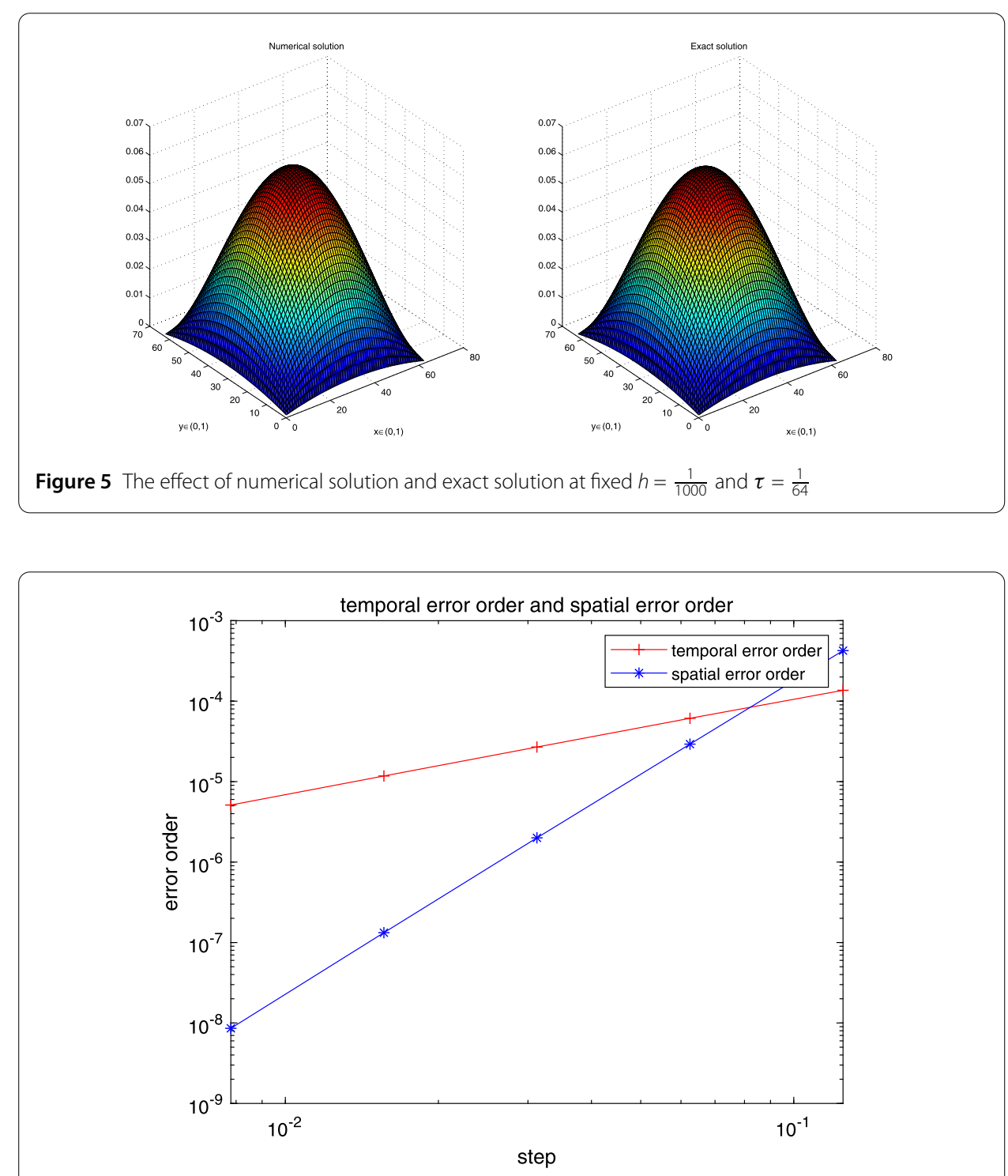

Figure 6 The red line represents temporal error order, the blue line represents spatial error order

\section{Conclusion}

In this article, we constructed a compact finite volume scheme for the multi-term time fractional sub-diffusion equation. We proved the existence and uniqueness, stability and $L_{\infty}$ convergence of our scheme. Two numerical results show that the scheme is conserved and convergent with the order $O\left(\tau^{2-\alpha}+h^{4}\right)$. 


\section{Acknowledgements}

The authors would like to express their sincere thanks to the referees for the valuable comments and suggestions which helped to improve the original paper.

\section{Funding}

This work is supported by the National Natural Science Foundation of China (No. 11171193) and the Natural Science Foundation of Shandong Province of China (No. ZR2017MA020).

\section{Availability of data and materials}

Not applicable.

\section{Competing interests}

The authors declare that they have no competing interests.

\section{Authors' contributions}

BS carried out the main part of this article. All authors read and approved the final manuscript.

\section{Publisher's Note}

Springer Nature remains neutral with regard to jurisdictional claims in published maps and institutional affiliations.

\section{Received: 23 January 2020 Accepted: 19 November 2020 Published online: 09 December 2020}

\section{References}

1. Agarwal, P., Baltaeva, U., Alikulov, Y.: Solvability of the boundary-value problem for a linear loaded integro-differential equation in an infinite three-dimensional domain. Chaos Solitons Fractals 140, 110108 (2020)

2. Agarwal, P., Waldschmidt, M., Kanemitsu, S., Jain, S.: Recent Trends in Special Functions and Analysis of Differential Equations. CRC Press, Boca Raton (2020)

3. Alderremy, A.A., Saad, K.M., Agarwal, P., et al.: Certain new models of the multi space-fractional Gardner equation. Phys. A, Stat. Mech. Appl. 545, 123806 (2020)

4. Bagley, R.L., Torvik, R.J.: On the appearance of the fractional derivative in the behaviour of real materials. Appl. Mech. 51, 294-298 (1984)

5. Baleanu, D., Defterli, O., Agrawal, O.P.: A central difference numerical scheme for fractional optimal control problems, J. Vib. Control 15(4), 583-597 (2009)

6. Benson, D.A., Wheatcraft, S.W., Meerschaert, M.M.: Application of a fractional advection dispersion equation. Water Resour. Res. 36, 1403-1412 (2000)

7. Chen, C.M., Liu, F.W., Turner, I., et al.: Numerical schemes and multivariate extrapolation of a two-dimensional anomalous sub-diffusion equation. Numer. Algorithms 54, 1-21 (2010)

8. Cui, M.: Finite difference schemes for the variable coefficients single and multi-term time-fractional diffusion equations with non-smooth solutions on graded and uniform meshes. Numer. Math., Theory Methods Appl. 12, 845-866 (2019)

9. Cui, M.: Compact difference scheme for time-fractional fourth-order equation with first Dirichlet boundary condition. East Asian J. Appl. Math. 9, 45-66 (2019)

10. Daftardar, G.V., Bhalekar, S.: Boundary value problems for multi-term fractional differential equations. J. Math. Anal. Appl. 345, 754-765 (2008)

11. El-Sayed, A.A., Baleanu, D., Agarwal, P.: A novel Jacobi operational matrix for numerical solution of multi-term variable-order fractional differential equations. J. Taibah Univ. Sci. 14(1), 963-974 (2020)

12. Ervin, V.J., Roop, J.P.: Variational formulation for the stationary fractional advection dispersion equation. Numer. Methods Partial Differ. Equ. 22, 558-576 (2006)

13. Gao, G.H., Liu, R.: A compact difference scheme for fourth-order temporal multi-term fractional wave equations and maximum error estimates. East Asian J. Appl. Math. 9, 703-722 (2019)

14. Gao, G.H., Sun, Z.Z.: Two alternating direction implicit difference schemes for solving the two-dimensional time distributed-order wave equations. J. Sci. Comput. 69(2), 506-531 (2016)

15. Gautschi, W.: Some elementary inequalities relating to the gamma and incomplete gamma function. J. Math. Phys. 38, 77-81 (2006)

16. Ji, C.C., Sun, Z.Z:: A high-order compact finite difference scheme for the fractional sub-diffusion equation. J. Sci. Comput. 64(3), 959-985 (2015)

17. Langlands, T.A.M., Henry, B.I.: The accuracy and stability of an implicit solution method for the fractional diffusion equation. J. Comput. Phys. 205(2), 719-736 (2005)

18. Lin, X.L., Michael, K.N., Sun, H.W.: A separable preconditioner for time-space fractional Caputo-Riesz diffusion equations. Numer. Math., Theory Methods Appl. 11, 827-853 (2018)

19. Liu, F.W., Anh, V., Turner, I.: Numerical solution of the space fractional Fokker-Planck equation. Comput. Appl. Math. $166,209-219(2004)$

20. Liu, F.W., Yang, C., Burrage, K.: Numerical method and analytical technique of the modified anomalous subdiffusion equation with a nonlinear source term. Comput. Appl. Math. 231, 160-176 (2009)

21. Liu, F.W., Zhuang, P., Anh, V., Turner, I., Burrage, K.: Stability and convergence of the difference methods for the space-time fractional advection-diffusion equation. Appl. Math. Comput. 191, 12-20 (2007)

22. Liu, Q., Liu, F.W., Turner, I., et al.: Finite element approximation for a modified anomalous sub-diffusion equation. Appl. Math. Model. 35, 4103-4116 (2011)

23. Luchko, Y.: Initial-boundary-value problems for the generalized multi-term time fractional diffusion equation. J. Math. Anal. Appl. 374, 538-548 (2011)

24. Machado, J.: Analysis and Design of Fractional-Order Digital Control Systems. Gordon \& Breach, New York (1997) 
25. Mainardi, F., Raberto, M., Gorenflo, R., Scalas, E.: Fractional calculus and continuous-time finance II: the waiting-time distribution. Physica A 287, 468-481 (2000)

26. Nguyen, T., Au, V.V., Long, L.D., Baleanu, D.: Regularization of a terminal value problem for time fractional diffusion equation. Math. Methods Appl. Sci. 43(6), 3850-3878 (2020)

27. Oldham, K.B., Spanier, J.: Theory and Application of Differentiation and Integration to Arbitrary Order. Academic Press, San Diego (1974)

28. Oustaloup, A.: Commande Robuste d'Ordre Non Entier. Hermes (1991)

29. Podlubny, I.: Fractional Differential Equations. Academic Press, San Diego (1999)

30. Ren, J.C., Sun, Z.Z.: Efficient and stable numerical methods for multi-term time fractional sub-diffusion equations. East Asian J. Appl. Math. 4, 242-266 (2014)

31. Rui, H.X:: A conservative characteristic finite volume element method for solution of the advection-diffusion equation. Comput. Methods Appl. Mech. Eng. 197(45-48), 3862-3869 (2008)

32. Samko, S.G., Kilbas, A.A., Marichev, O.I.: Fractional Integrals and Derivatives: Theory and Applications. Gordon \& Breach, New York (1993)

33. Sheng, C.T., Shen, J.: A space-time Petrov-Galerkin spectral method for time fractional diffusion equation. Numer. Math., Theory Methods Appl. 11, 854-876 (2018)

34. Sun, H., Sun, Z.Z., Du, R.: A linearized second-order difference scheme for the nonlinear time-fractional fourth-order reaction-diffusion equation. Numer. Math., Theory Methods Appl. 12, 1168-1190 (2019)

35. Sun, Z.Z: Numerical Methods of Partial Differential Equations. Science Press, Beijing (2012)

36. Tadjeran, C., Meerschaert, M.M., Scheffler, H.-P.: A second-order accurate numerical approximation for the fractional diffusion equation. Comput. Phys. 213, 205-213 (2006)

37. Tang, T., Yu, H.J., Zhou, T.: On energy dissipation theory and numerical stability for time-fractional phase field equations. SIAM J. Sci. Comput. 41(6), A3757-A3778 (2019)

38. Tuan, N.H., Baleanu, D. Thach, T.N., et al.: Final value problem for nonlinear time fractional reaction-diffusion equation with discrete data. J. Comput. Appl. Math. 376, 112883 (2020)

39. Tuan, N.H., Huynh, L.N., Baleanu, D., Can, N.H.: On a terminal value problem for a generalization of the fractional diffusion equation with hyper-Bessel operator. Math. Methods Appl. Sci. 43(6), 2858-2882 (2020)

40. Tuan, N.H., Tuan, N.H., Baleanu, D., Thach, T.N.: On a backward problem for fractional diffusion equation with Riemann-Liouville derivative. Math. Methods Appl. Sci. 43(3), 1292-1312 (2020)

41. Wang, N.L., Agarwal, P., Kanemitsu, S.: Limiting values and functional and difference equations. Mathematics 8(3), 407 (2020)

42. Wang, T.K.: An altering direction finite volume element method for a class of two-dimensional viscous wave equations. J. Numer. Methods Comput. Appl. 45, 205-210 (2018)

43. Wang, Y.M., Wang, T.: Error analysis of a high-order compact ADI method for two-dimensional fractional convection-subdiffusion equations. Calcolo 53, 1-30 (2015)

44. Wang, Z.B., Vong, S.: Compact difference schemes for the modified anomalous fractional sub-diffusion equation and the fractional diffusion-wave equation. J. Comput. Phys. 277, 1-15 (2014)

45. Wei, Y.B., Zhao, Y.M., et al:: Spatial high accuracy analysis of FEM for two-dimensional multi-term time-fractional diffusion-wave equations. Acta Math. Appl. Sin. Engl. Ser. 34, 828-841 (2018)

46. Wu, S.L., Zhou, T.: Parareal algorithms with local time-integrators for time fractional differential equations. J. Comput. Phys. 358, 135-149 (2018)

47. Yang, S.P., Xiao, A.G., Su, H.: Convergence of the variational iteration method for solving multi-order fractional differential equations. Comput. Math. Appl. 60, 2871-2879 (2010)

48. Yuste, S.B., Acedo, L.: An explicit finite difference method and a new von Neumann-type stability analysis for fractional diffusion equations. SIAM J. Numer. Anal. 42(5), 1862-1874 (2005)

49. Zhang, K.R., Jiang, Z.W.: Compact finite volume method for one-dimensional Schrodinger equation. J. Shandong Norm. Univ. 34, 201-205 (2018)

50. Zhang, Y., Sun, Z.Z:: Alternating direction implicit schemes for the two-dimensional fractional sub-diffusion equation. J. Comput. Phys. 230, 8713-8728 (2015)

51. Zhang, Y., Sun, Z.Z., Wu, H.W.: Error estimates of Crank-Nicolson-type difference schemes for the sub-diffusion equation. SIAM J. Numer. Anal. 49, 2302-2322 (2011)

52. Zhou, J., Xu, D., Chen, H.B.: A weak Galerkin finite element method for multi-term time-fractional diffusion equations. East Asian J. Appl. Math. 8, 181-193 (2018)

53. Zhuang, P., Liu, F.W., Anh, V., Turner, I.: New numerical methods for the time fractional sub-diffusion equation. SIAM J. Numer. Anal. 46(2), 1079-1095 (2008)

54. Zhuang, P., Liu, F.W., Anh, V., Turner, l.: Stability and convergence of an implicit numerical method for the non-linear fractional reaction-subdiffusion process. IMA J. Appl. Math. 74, 645-667 (2009) 